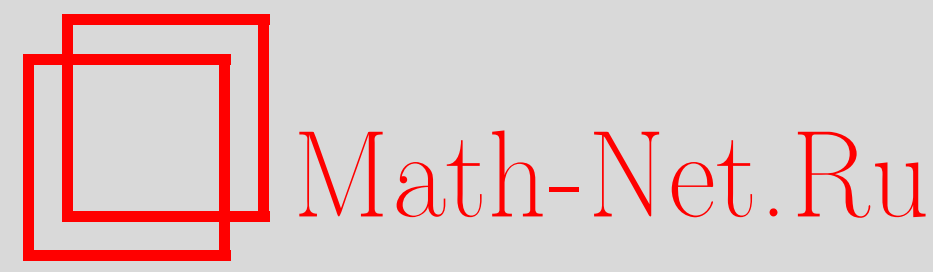

Е. Ю. Панов, К теории обобщенных энтропийных решений задачи Коши для одного класса нестрого гиперболических систем законов сохранения, Матем. сб., 2000, том 191, номер 1, 127-157

DOI: https://doi.org/10.4213/sm450

Использование Общероссийского математического портала Math-Net.Ru подразумевает, что вы прочитали и согласны с пользовательским соглашением

http://www.mathnet.ru/rus/agreement

Параметры загрузки:

IP: 54.162 .127 .20

26 апреля 2023 г., 15:09:48 


\author{
Е.Ю. Панов
}

\title{
К теории обобщенных энтропийных решений задачи Коши для одного класса нестрого гиперболических систем законов сохранения
}

Рассмотрены многомерные нестрого гиперболические системы законов сохранения с радиально вырожденным потоком. Для таких систем определено и описано множество энтропий, введено понятие обобщенного энтропийного решения (о.э.р.) задачи Коши, исследованы свойства о.э.р. Выделен класс сильных о.э.р., в котором рассматриваемая задача Коши однозначно разрешима. Приведено условие на начальные данные, при котором о.э.р. всегда является сильньм и, тем самым, единственным. При этом условии установлена сходимость метода "исчезающей вязкости". Примером показано, что в общем случае о.э.р. может быть неединственным.

Библиографоия: 18 названий.

\section{Введение}

В полуплоскости $\Pi=\mathbb{R}_{+} \times \mathbb{R}, \mathbb{R}_{+}=(0,+\infty)$, рассмотрим задачу Коши для системы

$$
\begin{gathered}
u_{t}+(\varphi(|u|) u)_{x}=0, \\
u=u(t, x) \in \mathbb{R}^{n}, \quad|u|=\left(u_{1}^{2}+\cdots+u_{n}^{2}\right)^{1 / 2},
\end{gathered}
$$

с начальным условием

$$
u(0, x)=u_{0}(x) \in L^{\infty}\left(\mathbb{R}, \mathbb{R}^{n}\right) .
$$

Предполагается, что функция $\varphi(r) \in C\left(\mathbb{R}_{+}\right)$и

$$
r \varphi(r) \underset{r \rightarrow 0+}{\longrightarrow} 0 .
$$

Системы вида (1) возникают в магнитогидродинамике при рассмотрении изотропной модели Кейфица-Кранзера.

В настоящей работе исследуются обобшенные энтропийные решения (о.э.р.) задачи Коши (1), (2). В 11 описывается множество допустимых энтропий. В $\S 2$ определяется понятие о.э.р. и исследуются свойства устойчивости о.э.р. В 33 выделяется класс сильных о.э.р., доказывается сушествование и единственность сильного о.э.р., приводится условие, при котором любое о.э.р. является сильным и, тем самьг, единственным. При этом условии доказывается также сходимость метода "исчезающей вязкости", чему посвящен $\S 4$.

Работа вьполнена при финансовой поддержке Российского фонда фундаментальных исследований (грант № 97-01-00671).

(C) Е. Ю. ПАнов 2000 
Изложение будет следовать, в основном, схеме работ [1], [2], в которых аналогичные результаты получены для систем вида

$$
U_{t}+\tilde{f}(U)_{x}=0
$$

$U=U(t, x) \in X$, где $X-$ пространство симметричных (или эрмитовых) матриц, $\tilde{f}(U)$ - функция от матрицы $U$, определенная в соответствии с функциональным исчислением по некоторой скалярной вещественной функции $f(u) \in C(\mathbb{R})$. В определенном смысле (см. замечание 3 ниже) система (1) представляет собой частный случай системы (4).

Для исследования (1) предположим сначала, что функция

$$
f(z)=\varphi(|z|) z \in C^{1}(\mathbb{R})
$$

здесь $f(0)=0$ в соответствии с условием $(3)$.

Тогда отображение $F(u)=\varphi(|u|) u C^{1}$-гладко на $\mathbb{R}^{n}$ и система (1) может быть переписана в виде

$$
u_{t}+A(u) u_{x}=0,
$$

где $A(u)=d F(u)$ - матрица с компонентами

$$
A_{i j}(u)=\varphi(|u|) \delta_{i j}+\varphi^{\prime}(|u|) \frac{u_{i} u_{j}}{|u|}, \quad i, j=1, \ldots, n,
$$

$\delta_{i j}=\left\{\begin{array}{ll}1, & i=j, \\ 0, & i \neq j,\end{array}\right.$-символы Кронекера.

Заметим, что $r \varphi^{\prime}(r)=f^{\prime}(r)-f(r) / r \underset{r \rightarrow 0+}{\longrightarrow} 0$ ввиду равенства $\varphi(r)=f(r) / r$, $r>0$, и условия (5). Поэтому

$$
\varphi^{\prime}(|u|) \frac{u_{i} u_{j}}{|u|}=\varphi^{\prime}(|u|)|u| \frac{u_{i}}{|u|} \frac{u_{j}}{|u|} \underset{u \rightarrow 0}{\longrightarrow} 0
$$

и $A_{i j}(0)=\varphi(0) \delta_{i j}$, где $\varphi(0)=\lim _{r \rightarrow 0} f(r) / r=f^{\prime}(0)$.

Ввиду симметричности матрицы $A(u)$ система $(1)-\left(1^{\prime}\right)$ - гиперболическая в широком смысле, т.е. (см. [3; гл. 1]) все собственные числа $A(u)$ вешественны и им соответствует полный набор собственных векторов. Легко видеть, что собственные числа матрицы $A(u)$ (с учетом кратности): $\lambda_{i}=\varphi(|u|)$, $i=1, \ldots, n-1, \lambda_{n}=\varphi(|u|)+\varphi^{\prime}(|u|)|u|$. Наличие совпадающих собственных значений означает, что система $(1)-\left(1^{\prime}\right)$ не является строго гиперболической по Лаксу (см. [3; гл. 1], [4]).

\section{§1. Энтропии}

Напомним (см. [5], [6]), что энтропией системы (1)-(1') называется функция $p(u) \in C^{1}\left(\mathbb{R}^{n}\right)$, для которой существует функция $q(u) \in C^{1}\left(\mathbb{R}^{n}\right)$, называемая потоком энтропии, такая, что для всех $u \in \mathbb{R}^{n}$

$$
\nabla q(u)=A(u) \nabla p(u)
$$


Ясно, что (7) определяет поток $q(u)$ с точностью до аддитивной константы.

Для наших целей будет полезно ослабить требование гладкости в определении энтропии. Обозначим через $\mathscr{F}$ множество функций $p(u) \in C^{1}\left(\mathbb{R}^{n} \backslash\{0\}\right)$, которые удовлетворяют условию Липшица в окрестности нуля. Доопределим градиент $\nabla p(u)$ функции $p(u) \in \mathscr{F}$ в нуле, положив $\nabla p(0)=0$.

ОПРЕДЕЛЕНИЕ 1. Функция $p(u) \in \mathscr{F}$ называется әнтропией системы (1), если найдется функция $q(u) \in \mathscr{F}$ (поток энтропиu) такая, что для всех $u \in \mathbb{R}^{n}$ вьполнено условие (7).

Поскольку при $p, q \in \mathscr{F}$ мы полагаем $\nabla p(0)=\nabla q(0)=0$, то в определении 1 достаточно требовать, чтобы (7) было выполнено при $u \neq 0$. Пусть $(\cdot, \cdot)-$ скалярное произведение в $\mathbb{R}^{n}$. Нам понадобится

Лемма 1. Пусть $u=u(t, x) \in C^{1}\left(\Pi, \mathbb{R}^{n}\right), p(u) \in \mathscr{F}$. Тогда

1) множество

$$
\mathscr{M}=\left\{(t, x) \in \Pi: u(t, x)=0,\left|u_{t}(t, x)\right|+\left|u_{x}(t, x)\right| \neq 0\right\}
$$

имеет нулевую меру Лебега (напомним, что |·| - евклидова норма в $\left.\mathbb{R}^{n}\right)$;

2) выполнены формуль дифференцирования суперпозиции

$$
p(u)_{t}=\left(\nabla p(u), u_{t}\right), \quad p(u)_{x}=\left(\nabla p(u), u_{x}\right)
$$

в пространстве распределений на П (в $\left.\mathscr{D}^{\prime}(\Pi)\right)$.

ДокаЗАТЕльство. 1) Если $(t, x) \in \mathscr{M}$, то векторы $u_{t}(t, x), u_{x}(t, x)$ не равны нулевому вектору одновременно и, значит, сушествует гиперплоскость $H \subset \mathbb{R}^{n}$ такая, что отображение $u(t, x)$ трансверсально к $H$ в некоторой окрестности $V$ точки $(t, x)$. Отсюда следует (см., например, [7; теорема 3.3]), что множество $V \cap u^{-1}(H)$ является подмногообразием коразмерности 1 и, в частности, множество $V \cap M \subset V \cap u^{-1}(H)$ имеет нулевую меру Лебега. Покрывая $\mathscr{M}$ счетным семейством таких окрестностей $V$ (что можно сделать благодаря 2-й аксиоме счетности), получаем, что $\mathscr{M}$ имеет нулевую меру Лебега.

2 ) По определению класса $\mathscr{F}$ функция $p(u)$ удовлетворяет локально на $\mathbb{R}^{n}$ условию Липшица, откуда следует, что суперпозиция $p(u(t, x))$ локально липшицева на П и обобщенные производные $p(u)_{t}, p(u)_{x}$ являются локально ограниченными функциями. При этом $p(u)_{t}, p(u)_{x}$ совпадают почти всюду с классическими частньми производными, которые существуют для почти всех $(t, x)$ (известно, что $p(u(t, x))$ дифференцируема в точках Лебега вектор-функции $(t, x) \mapsto\left(p(u)_{t}(t, x)\right.$, $\left.\left.p(u)_{x}(t, x)\right)\right)$. Таким образом, достаточно установить, что (8) вьполнено для классических производных на некотором множестве полной меры Лебега. Покажем, что при $(t, x) \notin \mathscr{M}$ функция $p(u(t, x))$ дифференцируема и справедливо (8). Так как $\mathscr{M}$ имеет нулевую меру, то это завершит доказательство. Для $(t, x) \notin \mathscr{M}$ возможны два случая:

a) $u(t, x) \neq 0$,

б) $u(t, x)=u_{t}(t, x)=u_{x}(t, x)=0$. 
В случае а) $p(u)$ дифференцируема при $u=u(t, x)$. По классической теореме о дифференцируемости суперпозиции $p(u(t, x))$ дифференцируема в точке $(t, x)$ и выполнены равенства (8). Рассмотрим случай б). По условию $u(t, x)=u_{t}(t, x)=$ $u_{x}(t, x)=0$, откуда

$$
|u(t+\Delta t, x+\Delta x)|=o(|\Delta t|+|\Delta x|) \text { при }|\Delta t|+|\Delta x| \rightarrow 0 .
$$

Из локальной липшицевости функции $p(u)$ и $(9)$ следует, что существует константа $k>0$ такая, что при достаточно малых $\Delta t, \Delta x$

$$
|p(u(t+\Delta t, x+\Delta x))-p(0)| \leqslant k|u(t+\Delta t, x+\Delta x)|=o(|\Delta t|+|\Delta x|)
$$

Поэтому $p(u)_{t}(t, x)=p(u)_{x}(t, x)=0$ и равенства (8) также выполнены. Лемма полностью доказана.

Если $u=u(t, x)-C^{1}$-гладкое решение системы $(1), p(u)$ - энтропия, а $q(u)$ соответствующий поток, то, умножая систему $\left(1^{\prime}\right)$ скалярно на $\nabla p(u)$ и учитывая симметричность матрицы $A(u)$, равенство $(7)$ и формулы $(8)$, получаем, что в $\mathscr{D}^{\prime}(\Pi)$

$$
\begin{aligned}
0 & =\left(\nabla p(u), u_{t}\right)+\left(\nabla p(u), A(u) u_{x}\right)=\left(\nabla p(u), u_{t}\right)+\left(A(u) \nabla p(u), u_{x}\right) \\
& =\left(\nabla p(u), u_{t}\right)+\left(\nabla q(u), u_{x}\right)=(p(u))_{t}+(q(u))_{x}
\end{aligned}
$$

Итак,

$$
p(u)_{t}+q(u)_{x}=0 \quad \text { в } \mathscr{D}^{\prime}(\Pi)
$$

для любой энтропии $p(u)$ и соответствуюшего потока $q(u)$. Заметим, что для гладких энтропий равенство (10) выполнено в классическом смысле и хорошо известно.

Опишем множество энтропий системы (1). Прежде всего заметим, что ввиду (6) равенство (7) можно переписать в виде

$$
\begin{aligned}
\nabla q(u)=A(u) \nabla p(u) & =\varphi(r) \nabla p(u)+\varphi^{\prime}(r)\left(\nabla p(u), \frac{u}{r}\right) u \\
& =\varphi(r) \nabla p(u)+\varphi^{\prime}(r) p_{r}(u) u
\end{aligned}
$$

где $r=|u|, p_{r}(u)=(\nabla p(u), u / r)$ - радиальная производная.

Справедлива

Теорема 1. Пусть функиия $\eta(r) \in C^{1}\left(\mathbb{R}_{+}\right)$и непрерывна по Липиицу в окрестности нуля, $h(u) \in C^{1}\left(\mathbb{R}^{n} \backslash\{0\}\right)$ - однородная (степени 1) функиия: $h(r u)=r h(u) \forall r>0$. Тогда

$$
p(u)=\eta(|u|)+h(u)
$$

является энтропией системы (1) с потоком

$$
q(u)=\psi(|u|)+\varphi(|u|) h(u)
$$

где $\psi(r)$ определена с точностью до аддитивной константы равенством $\psi^{\prime}(r)=\eta^{\prime}(r) f^{\prime}(r), r>0$. 
ДокаЗАТЕЛьство. Достаточно проверить равенство (11) при $r=|u|>0$ для энтропий $p=p_{1}, h$ и соответствующих потоков $q=q_{1}, q_{2}$, где $p_{1}=\eta(r)$, $q_{1}=\psi(r), q_{2}=\varphi(r) h(u)$. Легко видеть, что функция $\psi(r) \in C^{1}\left(\mathbb{R}_{+}\right)$и непрерьвна по Липшицу в окрестности нуля, как и $\eta(r)$. Поэтому $p_{1}, q_{1} \in \mathscr{F}$. Далее, при $r=|u|>0 \nabla p_{1}(u)=\eta^{\prime}(r) u / r, \nabla q_{1}(u)=\psi^{\prime}(r) u / r=\eta^{\prime}(r) f^{\prime}(r) u / r$ и

$$
A(u) \nabla p_{1}(u)=\eta^{\prime}(r)\left(\varphi(r)+r \varphi^{\prime}(r)\right) \frac{u}{r}=\eta^{\prime}(r) f^{\prime}(r) \frac{u}{r}=\nabla q_{1}(u)
$$

т.е. вьполнено соотношение (11).

Включения $h, q_{2} \in \mathscr{F}$ очевидны. Заметим, что в силу однородности $h(u)=$ $r h(\xi), q_{2}(u)=f(r) h(\xi)$, где $r=|u|, \xi=u /|u| \in S, S=S^{n-1}$ - единичная сфера в $\mathbb{R}^{n}$. Ввиду равенства $h_{r}(u)=h(\xi)=h(u) / r$ имеем при $r>0$

$$
\begin{aligned}
A(u) \nabla h(u) & =\varphi(r) \nabla h(u)+\varphi^{\prime}(r) h_{r}(u) u=\varphi(r) \nabla h(u)+h(u) \varphi^{\prime}(r) \frac{u}{r} \\
& =\varphi(r) \nabla h(u)+h(u) \nabla \varphi(r)=\nabla(\varphi(r) h(u))=\nabla q_{2}(u),
\end{aligned}
$$

и соотношение (11) также выполнено. Теорема полностью доказана.

Энтропии вида (12) мы будем называть допустимыми. Оказывается, в достаточно общей ситуации любая энтропия системы (1) является допустимой. Точнее, справедлива

ТЕОРема 2. Предположим, что $\varphi^{\prime}(r) \neq 0$ на всюду плотном множестве значений $r \in \mathbb{R}_{+}$. Тогда любая энтропия $p(u)$ системь (1) является допустимой.

ДОКАЗАТЕЛЬСтво. Пусть $q(u)$ - поток, соответствующий энтропии $p(u)$. Введем сферические координаты: $r=|u|, \xi=u / r \in S, S$ - единичная сфера в $\mathbb{R}^{n}$. Тогда $u=r \xi$ и из (11) вытекают равенства

$$
\begin{gathered}
q_{r}(u)=\left(\varphi(r)+\varphi^{\prime}(r) r\right) p_{r}(u)=f^{\prime}(r) p_{r}(u), \\
\nabla_{\xi} q(u)=\varphi(r) \nabla_{\xi} p(u),
\end{gathered}
$$

$\nabla_{\xi}$ - градиент на сфере $\xi \in S$ при фиксированном $r$. Из (15) сразу следует, что $q(u)=\varphi(r) p(u)+c(r)$, где $c(r)$ - некоторая гладкая функция на $\mathbb{R}_{+}$. Подставляя это выражение в (14), получаем, что

$$
\varphi^{\prime}(r) p(u)+\varphi(r) p_{r}(u)+c^{\prime}(r)=\left(\varphi(r)+\varphi^{\prime}(r) r\right) p_{r}(u)
$$

откуда $\varphi^{\prime}(r)\left(r p_{r}(u)-p(u)\right)=c^{\prime}(r)$ и при $\varphi^{\prime}(r) \neq 0$ функция $r p_{r}(u)-p(u)$ не зависит от $\xi$. По условию множество таких $r$ плотно в $\mathbb{R}_{+}$. Поэтому $r p_{r}(u)-p(u)$ не зависит от $\xi$ при всех $r>0$ и для некоторой непрерывной на $\mathbb{R}_{+}$функции $\gamma(r)$

$$
r^{2}\left(\frac{p(u)}{r}\right)_{r}=r p_{r}(u)-p(u)=\gamma(r)
$$


Решение последнего уравнения имеет вид $p(u)=\eta(r)+r \alpha(\xi)$, где ввиду условия $p(u) \in \mathscr{F}$ функция $\eta(r) \in C^{1}\left(\mathbb{R}_{+}\right)$и удовлетворяет условию Липшица в окрестности нуля, $\alpha(\xi) \in C^{1}(S)$.

Поскольку $h(u)=r \alpha(\xi) \in C^{1}\left(\mathbb{R}^{n} \backslash\{0\}\right)$ - однородная степени 1 функция, то $p(u)$ имеет вид (12). Теорема доказана.

Отметим, что допустимая энтропия является гладкой на $\mathbb{R}^{n}$ тогда и только тогда, когда найдется представление $p(u)=\eta(|u|)+h(u)$, в котором $\eta(r) \in C^{1}[0,+\infty)$, $\eta^{\prime}(0)=0$, a $h(u)$ - линейная функция.

Нам понадобятся также следующие условия выпуклости допустимых энтропий.

ПРЕДЛОЖЕНИЕ 1. 1) Предположим, что допустимая әнтропия вида (12) выпукла на $\mathbb{R}^{n}$. Тогда функция $\eta(r)$ выпукла на $\mathbb{R}_{+}$.

2) Энтропия вида $p(u)=\eta(|u|)$ выпукла на $\mathbb{R}^{n}$ тогда и только тогда, когда функиия $\eta(r)$ выпукла и не убывает на $\mathbb{R}_{+}$.

3) Однородная әнтропия $p(u)=h(u)$ выпукла на $\mathbb{R}^{n}$ тогда и только тогда, когда функция $h(u)$ сублинейна (т.е. в дополнение к однородности выполнено свойство субаддитивности $\left.h(u+v) \leqslant h(u)+h(v) \quad \forall u, v \in \mathbb{R}^{n}\right)$.

ДокАЗАТЕЛьство. 1) Пусть $p(u)=\eta(|u|)+h(u)-$ выпуклая функция, $e \in \mathbb{R}^{n}$ - вектор единичной длины. Тогда функция $g(r)=p(r e)=\eta(r)+h(e) r$ выпукла при $r>0$, откуда и вытекает выпуклость $\eta(r)$.

2) Если энтропия $p(u)=\eta(|u|)$ выпукла на $\mathbb{R}^{n}$, то в обозначениях п. 1) функция $p(r e)=\eta(|r|)$ выпукла и четна на $\mathbb{R}$, откуда непосредственно следует, что $\eta(r)$ вьпукла и не убывает на $\mathbb{R}_{+}$. Обратно, если функция $\eta(r)$ выпукла и не убывает на $\mathbb{R}_{+}$, то $p(u)=\eta(|u|)$ выпукла на $\mathbb{R}^{n}$ как композиция функции $\eta(r)$ и выпуклой на $\mathbb{R}^{n}$ функции $|u|$.

3) Последнее утверждение очевидно, ибо для однородных функций условие выпуклости эквивалентно условию субаддитивности.

\section{§2. Обобщенные энтропийные решения}

Пусть начальная вектор-функция $u_{0} \in L^{\infty}\left(\mathbb{R}, \mathbb{R}^{n}\right)$. Определим понятие о.э.р. задачи (1), (2).

ОПРЕДЕЛЕНИЕ 2. Ограниченная измеримая вектор-функция $u=u(t, x) \in$ $L^{\infty}\left(\Pi, \mathbb{R}^{n}\right)$ называется обобщенным энтропийным решением (о.э.р.) задачи Коши (1), (2), если

а) для любой допустимой выпуклой энтропии $p(u) \in \mathscr{F}$ и соответствующего потока $q(u)$ выполнено энтропийное условие Кружкова-Лакса (см. [5], [6]):

$$
p(u)_{t}+q(u)_{x} \leqslant 0 \quad \text { в } \mathscr{D}^{\prime}(\Pi)
$$

b)

$$
\underset{t \rightarrow 0+}{\operatorname{ess}} \lim u(t, \cdot)=u_{0} \quad \text { в } \quad L_{\mathrm{loc}}^{1}\left(\mathbb{R}, \mathbb{R}^{n}\right),
$$

т.е. существует множество нулевой меры Лебега $\mathscr{E} \subset \mathbb{R}_{+}$такое, что $u(t, \cdot) \in$ $L_{\text {loc }}^{1}\left(\mathbb{R}, \mathbb{R}^{n}\right), t \notin \mathscr{E}$, и $u(t, \cdot) \rightarrow u_{0}$ в $L_{\text {lос }}^{1}\left(\mathbb{R}, \mathbb{R}^{n}\right)$ при $t \rightarrow 0+, t \notin \mathscr{E}$. 
Условие а) означает, что для любой неотрицательной пробной функции $g=$ $g(t, x) \in C_{0}^{\infty}(\Pi)$

$$
\int_{\Pi}\left[p(u) g_{t}+q(u) g_{x}\right] d t d x \geqslant 0 .
$$

Заметим, что ввиду (10) для классического решения $u(t, x) \in C^{1}\left(\Pi, \mathbb{R}^{n}\right)$ условие (16) выполнено со знаком равенства.

ЗАмЕчАниЕ 1 . Подставляя в (16) линейные энтропии $p(u)= \pm(c, u), c \in \mathbb{R}^{n}$ (которые допустимы ввиду однородности), получаем, что о.э.p. $u(t, x)$ удовлетворяет системе (1) в смысле распределений, т.е. действительно является обобщенным решением.

ЗАмЕчАниЕ 2 . Если $T$ - ортогональное преобразование на $\mathbb{R}^{n}$, то функции $p(T u), q(T u)$ образуют пару, состоящую из выпуклой допустимой энтропии и соответствующего ей потока, одновременно с функциями $p(u), q(u)$. Отсюда легко следует, что для о.э.р. $u$ задачи (1), (2) вектор-функция $T u$ также является о.э.р. этой задачи с начальными данньми $T u_{0}$.

Для общих гиперболических систем известно (см. [5]), что определяющее энтропийное условие Кружкова-Лакса (16) с гладкими энтропиями $p(u)$ вытекает из метода “исчезающей вязкости”. Покажем, что это остается справедливым и для энтропий из класса $\mathscr{F}$. Рассмотрим параболическую аппроксимацию системы (1):

$$
u_{t}+(\varphi(|u|) u)_{x}=u_{t}+A(u) u_{x}=\varepsilon u_{x x}, \quad \varepsilon>0
$$

ПРЕДЛОЖЕНИЕ 2. Пусть существует последовательность $\varepsilon_{k} \rightarrow 0$ такая, что определено решение $u^{k}=u^{k}(t, x) \in C^{2}\left(\Pi, \mathbb{R}^{n}\right)$ системы $(18)$ с $\varepsilon=\varepsilon_{k} u$ при $k \rightarrow \infty$ последовательность $u^{k}$ сходится в $L_{\mathrm{loc}}^{1}\left(\Pi, \mathbb{R}^{n}\right) \kappa$ вектор-функиии $u=u(t, x)$. Тогда $u(t, x)$ удовлетворяет условию (16) для любой выпуклой энтропии $p(u) \in \mathscr{F}$ и соответствующего потока $q(u)$.

Для доказательства нам понадобится следующая

Лемма 2. 1) Пусть $p(u) \in \mathscr{F}$ - выпуклая функиия на $\mathbb{R}^{n}, u=u(t, x) \in$ $C^{2}\left(\Pi, \mathbb{R}^{n}\right)$. Тогда $\left(\nabla p(u), u_{x x}\right) \leqslant p(u)_{x x}$ в $\mathscr{D}^{\prime}(\Pi)$.

2) Если $u=u(t, x) \in C^{2}\left(\Pi, \mathbb{R}^{n}\right)$ - решение системы (18), то для любой выпуклой энтропии $p(u) \in \mathscr{F}$ и соответствующего потока $q(u)$ справедливо соотношение $p(u)_{t}+q(u)_{x} \leqslant \varepsilon p(u)_{x x}$ в $\mathscr{D}^{\prime}(\Pi)$.

ДокАЗАТЕЛьСтво. При $R>0$ через $B_{R}=\left\{u \in \mathbb{R}^{n}:|u| \leqslant R\right\}$ обозначим шар радиуса $R$ в $\mathbb{R}^{n}$. Пусть $\rho(u) \in C_{0}^{\infty}\left(\mathbb{R}^{n}\right), \rho(u) \geqslant 0, \operatorname{supp} \rho \subset B_{1}, \int \rho(u) d u=1$. При $k \in \mathbb{N}$ положим $\rho_{k}(u)=k^{n} \rho(k u)$. Тогда $\rho_{k}(u) \in C_{0}^{\infty}\left(\mathbb{R}^{n}\right), \rho_{k}(u) \geqslant 0$, $\operatorname{supp} \rho_{k} \subset B_{1 / k}, \int \rho_{k}(u) d u=1$. Из приведенных свойств следует, что при $k \rightarrow \infty$ последовательность $\rho_{k}(u)$ сходится в $\mathscr{D}^{\prime}\left(\mathbb{R}^{n}\right)$ к $\delta$-функции Дирака. Рассмотрим средние функции

$$
p_{k}(u)=\left(p * \rho_{k}\right)(u)=\int_{\mathbb{R}^{n}} p(u-v) \rho_{k}(v) d v
$$


Из свойств средних функций следует, что для всех $k \in \mathbb{N} p_{k}(u)$ - выпуклая бесконечно дифференцируемая функция и (с учетом определения класса $\mathscr{F})$ при $k \rightarrow \infty$

$$
\begin{gathered}
p_{k}(u) \rightarrow p(u) \text { равномерно на компактах в } \mathbb{R}^{n} ; \\
\nabla p_{k}(u) \rightarrow \nabla p(u) \text { равномерно на компактах в } \mathbb{R}^{n} \backslash\{0\} .
\end{gathered}
$$

Пусть

$$
\mathscr{M}=\left\{(t, x) \in \Pi: u(t, x)=0, u_{x x}(t, x) \neq 0\right\} .
$$

Тогда $\mathscr{M} \subset \mathscr{M}_{1} \cup \mathscr{M}_{2}$, где

$$
\begin{aligned}
& \mathscr{M}_{1}=\left\{(t, x) \in \Pi: u(t, x)=0, u_{x}(t, x) \neq 0\right\} \\
& \mathscr{M}_{2}=\left\{(t, x) \in \Pi: u_{x}(t, x)=0, u_{x x}(t, x) \neq 0\right\} .
\end{aligned}
$$

Применяя лемму 1 к гладким функциям $u, u_{x}$, получаем, что множества $\mathscr{M}_{1}, \mathscr{M}_{2}$ имеют нулевую меру, откуда следует, что и $\mathscr{M}$ - множество нулевой меры. Покажем, что при $(t, x) \notin \mathscr{M}$ (т.е. для почти всех $(t, x))$

$$
\left(\nabla p_{k}(u), u_{x x}\right) \underset{k \rightarrow \infty}{\longrightarrow}\left(\nabla p(u), u_{x x}\right), \quad u=u(t, x)
$$

Для этого рассмотрим два возможных случая.

1) $u(t, x) \neq 0$. В этом случае $(21)$ непосредственно следует из предельного соотношения (20).

2) $u(t, x)=0, u_{x x}(t, x)=0$. Тогда $\left(\nabla p(u), u_{x x}\right)=\left(\nabla p_{k}(u), u_{x x}\right)=0$ и $(21)$ очевидно.

Далее, легко видеть, что

$$
\left(\nabla p_{k}(u), u_{x x}\right)=p_{k}(u)_{x x}-d^{2} p_{k}(u)\left(u_{x}, u_{x}\right)
$$

Ввиду вьпуклости $p_{k}$ форма $d^{2} p_{k}(u)(d u, d u)$ неотрицательна, и мы приходим к неравенству $\left(\nabla p_{k}(u), u_{x x}\right) \leqslant p_{k}(u)_{x x}$, умножая которое на неотрицательную пробную функцию $g=g(t, x) \in C_{0}^{\infty}(\Pi)$ и интегрируя на П с использованием формулы интегрирования по частям, получаем, что

$$
\int_{\Pi}\left(\nabla p_{k}(u), u_{x x}\right) g d t d x \leqslant \int_{\Pi} p_{k}(u) g_{x x} d t d x
$$

Перейдем в этом неравенстве к пределу при $k \rightarrow \infty$. По теореме Лебега об ограниченной сходимости (заметим, что с учетом локальной липшицевости $p(u)$ функции $\left(\nabla p_{k}(u), u_{x x}\right)$ ограничены равномерно по $k$ на любом компакте в П) из предельных соотношений (19), (21) следует, что

$$
\int_{\Pi}\left(\nabla p(u), u_{x x}\right) g d t d x \leqslant \int_{\Pi} p(u) g_{x x} d t d x
$$

откуда ввиду произвольности неотрицательной пробной функции $g=g(t, x) \in$ $C_{0}^{\infty}(\Pi)$ получим, что $\left(\nabla p(u), u_{x x}\right) \leqslant p(u)_{x x}$ в $\mathscr{D}^{\prime}(\Pi)$. Первое утверждение леммы доказано. Для доказательства второго утверждения предположим, что 
$u=u(t, x) \in C^{2}\left(\Pi, \mathbb{R}^{n}\right)$ - решение системы $(18), p(u) \in \mathscr{F}$ - выпуклая энтропия системы (1), а $q(u) \in \mathscr{F}$ - соответствуюший поток. Умножим равенство $u_{t}+A(u) u_{x}=\varepsilon u_{x x}$ на $\nabla p(u)$. Используя лемму 1 , равенство $(7)$ и уже доказанное первое утверждение леммы 2 , получаем требуемое соотношение

$$
\begin{aligned}
p(u)_{t}+q(u)_{x} & =\left(\nabla p(u), u_{t}\right)+\left(\nabla q(u), u_{x}\right)=\left(\nabla p(u), u_{t}\right)+\left(A(u) \nabla p(u), u_{x}\right) \\
& =\left(\nabla p(u), u_{t}+A(u) u_{x}\right)=\varepsilon\left(\nabla p(u), u_{x x}\right) \leqslant \varepsilon p(u)_{x x}
\end{aligned}
$$

в $\mathscr{D}^{\prime}(\Pi)$. Лемма доказана.

ДоКАЗАТЕЛЬСтво ПРЕДЛОЖЕНИЯ 2. По лемме 2 для всех $k \in \mathbb{N}$ выполнено неравенство

$$
p\left(u^{k}\right)_{t}+q\left(u^{k}\right)_{x} \leqslant \varepsilon_{k} p\left(u^{k}\right)_{x x} \quad \text { в } \mathscr{D}^{\prime}(\Pi),
$$

умножая которое на неотрицательную пробную функцию $g=g(t, x) \in C_{0}^{\infty}(\Pi)$ и интегрируя на П, получаем, что

$$
-\int_{\Pi}\left[p\left(u^{k}\right) g_{t}+q\left(u^{k}\right) g_{x}\right] d t d x \leqslant \varepsilon_{k} \int_{\Pi} p\left(u^{k}\right) g_{x x} d t d x
$$

Переходя здесь к пределу при $k \rightarrow \infty$ и принимая во внимание, что $\varepsilon_{k} \rightarrow 0$, получаем неравенство

$$
\int_{\Pi}\left[p(u) g_{t}+q(u) g_{x}\right] d t d x \geqslant 0
$$

эквивалентное (16) ввиду произвольности неотрицательной пробной функции $g=g(t, x) \in C_{0}^{\infty}(\Pi)$. Предложение доказано.

Заметим, что поток $q$ выпуклой допустимой энтропии определен по формуле (13) и непрерывен на $\mathbb{R}^{n}$ в случае лишш непрерывной функции $f(z)=\varphi(|z|) z$ (т.е. при вьполнении условия (3)), нужно только переопределить функцию $\psi(r)$, положив

$$
\psi(r)=\eta^{\prime}(r) f(r)-\int_{0}^{r} f(\sigma) d \eta^{\prime}(\sigma)
$$

(так что в гладком случае $f \in C^{1}$ верно тождество $\psi^{\prime}(r)=\eta^{\prime}(r) f^{\prime}(r)$ ). Здесь в соответствии с предложением 1 функция $\eta(r)$ выпукла на $\mathbb{R}_{+}$и $d \eta^{\prime}(\sigma)$ - неотрицательная борелевская мера.

Таким образом, определение 2 корректно и в общем случае, когда выполнено условие (3), что и будет всюду предполагаться. В этом обшем случае мы будем часто использовать следующие два технических результата, доказанные в [2].

ЛЕмма 3 (см. также [8]). Пусть $\omega: \mathbb{R}_{+} \mapsto \mathbb{R}_{+}-$неубывающая субаддитивная функиия. Тогда $\sup _{\sigma>0}\{\omega(\sigma) /(\sigma+\varepsilon)\} \leqslant \omega(\varepsilon) / \varepsilon \forall \varepsilon>0$. 
Лемма 4. Пусть $\alpha_{t}+\beta_{x} \leqslant 0$ в $\mathscr{D}^{\prime}(\Pi)$, әде $\alpha=\alpha(t, x), \beta=\beta(t, x) \in L^{\infty}(\Pi)$;

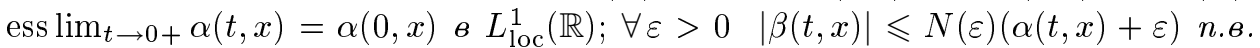
в П (из этого условия следует, что $\alpha \geqslant 0$ п.в. на П), $N(\varepsilon) \geqslant 1$. Тогда для почти всех $t>0$ справедлива оценка:

$$
\int \alpha(t, x) e^{-|x|} d x \leqslant e^{t} \cdot \inf _{\varepsilon>0}\left(\int \alpha(0, x) e^{-|x| / N(\varepsilon)} d x+2 \varepsilon N(\varepsilon)\right) .
$$

Ниже нам понадобятся понятия о.э.р. и обобщенного энтропийного суб-решения (коротко - о.э.суб-р.) задачи Коши для скалярного квазилинейного уравнения

$$
r_{t}+f(r)_{x}=0
$$

с начальным условием

$$
r(0, x)=r_{0}(x) \in L^{\infty}(\mathbb{R}) .
$$

ОПРЕДЕЛЕНИЕ 3 (см. [5], [8]-[10]). Функция $r=r(t, x) \in L^{\infty}(\Pi)$ называется обобщенным энтропийным решением (о.э.р.) задачи Коши (23), (24), если

а) для любой выпуклой функции $\eta \in C^{1}(\mathbb{R})$ (энтропии)

$$
\eta(r)_{t}+\psi(r)_{x} \leqslant 0 \quad \text { в } \mathscr{D}^{\prime}(\Pi)
$$

где функция $\psi(r)$ (поток) с точностью до аддитивной константы определена равенством $(22)$;

b) $\operatorname{ess~}_{\lim } \rightarrow 0+r(t, \cdot)=r_{0}$ в $L_{\mathrm{loc}}^{1}(\mathbb{R})$.

Подставляя в (25) линейные энтропии $\eta(r)= \pm r$, получаем, что о.э.p. $r(t, x)$ удовлетворяет уравнению (23) в смысле распределений. Заметим также (см. [5], [9]-[11]), что условие а) определения 3 эквивалентно известному условию С.Н. Кружкова из [5]:

$\left.\mathrm{a}^{\prime}\right) \forall k \in \mathbb{R}|r-k|_{t}+((f(r)-f(k)) \operatorname{sign}(r-k))_{x} \leqslant 0$ в $\mathscr{D}^{\prime}(\Pi)$.

Как установлено в [5], при $f(r) \in C^{1}(\mathbb{R})$ всегда сушествует единственное о.э.р. задачи Коши $(23),(24)$. При $f(r) \in C(\mathbb{R})$ о.э.р. также всегда существует, но может быть неединственньм в случае более одной пространственной переменной (см. [8], [12]). Однако, как показано в [8], [11], [12], для рассматриваемого случая одной пространственной переменной $x$ задача Коши всегда однозначно разрешима.

Обозначим $u^{+}=\max (u, 0), u^{-}=\max (-u, 0)$.

ОПРЕДЕЛЕНИЕ 4 (см. [8]-[10]). Функция $r=r(t, x) \in L^{\infty}(\Pi)$ назьвается обобщенным әнтропийным суб-решением (о.ә.суб.-р.) задачи Коши (23), (24), если для любой неубывающей выпуклой функции $\eta \in C^{1}(\mathbb{R})$ выполнено условие $(25)$ и ess $\lim _{t \rightarrow 0+}\left(r(t, \cdot)-r_{0}\right)^{+}=0$ в $L_{\text {loc }}^{1}(\mathbb{R})$.

Понятие обобшенного энтропийного супер-решения определяется аналогично, здесь нужно требовать выполнения условия (25) для невозрастающих выпуклых функций, а в начальном условии заменить $\left(r(t, \cdot)-r_{0}\right)^{+}$на $\left(r(t, \cdot)-r_{0}\right)^{-}$. Ясно, что функция $u$ - о.э.р. задачи $(23),(24)$ тогда и только тогда, когда $u$ одновременно является обобщенным энтропийным суб- и супер-решением этой задачи.

Нам понадобятся некоторые частные случаи принципа сравнения, установленного в работах [8]-[10]. 
ПРЕДЛОЖЕНИЕ 3. Пусть $r=r(t, x)-$ о.э.суб-р. задачи $(23),(24) u r_{0}(x) \leqslant M$ n.в. на $\mathbb{R}$ для некоторой константы $M$. Тогда $r(t, x) \leqslant M$ n.в. на П (принцип максимума). Если $r=r(t, x)$ - о.э.р. задачи (23), $(24) u a \leqslant r_{0}(x) \leqslant b$ n.в. на $\mathbb{R}$ для $a \leqslant b$, то $a \leqslant r(t, x) \leqslant b$ п.в. на П.

Если $\eta(r) \in C^{1}[0,+\infty)$ - неубываюшая выпуклая функция, то функция $p(u)=$ $\eta(|u|)$ является допустимой выпуклой энтропией системы (1) с потоком $q(u)=$ $\psi(|u|)$, где функция $\psi(r)$ определена соотношением (22). Из условий (16), (17) определения 2 тогда сразу следует

ТЕОРема 3. Если $u=u(t, x)$ - о.э.р. задачи $(1),(2)$, mo $r=r(t, x)=|u(t, x)|$ является о.э.суб-р. задачи Коши (23), (24) с начальной функичей $r_{0}(x)=$ $\left|u_{0}(x)\right|$ (причем выполнено строгое начальное условие в смысле требования b) определения 3$)$.

Из теоремы 3 и предложения 3 вытекает принцип максимума для о.э.р. исходной задачи (1), (2):

СЛЕДСТВИЕ 1. Пусть $\left|u_{0}(x)\right| \leqslant M$ п.в. на $\mathbb{R}$ для некоторой константы $M>0$. Тогда если $u(t, x)$ - о.э.р. задачи (1), (2), то $|u(t, x)| \leqslant M$ п.в. на П.

Пусть $e \in \mathbb{R}^{n}$ - вектор единичной длины, $0 \leqslant \delta \leqslant 1$. Рассмотрим конусы

$$
\begin{aligned}
& \Gamma(e, \delta)=\left\{u \in \mathbb{R}^{n}:(u, e) \geqslant \delta|u|\right\}, \\
& \dot{\Gamma}(e, \delta)=\left\{u \in \mathbb{R}^{n}:(u, e)>\delta|u|\right\} \cup\{0\} .
\end{aligned}
$$

Справедлива следующая

TEOPEMA 4. Пусть $u_{0}(x) \in \Gamma(e, \delta)\left(u_{0}(x) \in \dot{\Gamma}(e, \delta)\right)$ для почти всех $x \in \mathbb{R}$. Тогда если $u=u(t, x)$ - о.э.р. задачи (1), (2), то $u(t, x) \in \Gamma(e, \delta)$ (coответственно $u(t, x) \in \dot{\Gamma}(e, \delta))$ п.в. на П.

ДокАЗАТЕЛьство. Ввиду замечания 2 с помощью ортогонального преобразования вектора $u$ можно свести доказательство к случаю, когда $e=(1,0, \ldots, 0)-$ первый базисньй вектор. В этом случае

$$
\begin{aligned}
& \Gamma(e, \delta)=\Gamma(\delta)=\left\{u \in \mathbb{R}^{n}: u_{1} \geqslant \delta|u|\right\} \\
& \dot{\Gamma}(e, \delta)=\dot{\Gamma}(\delta)=\left\{u \in \mathbb{R}^{n}: u_{1}>\delta|u|\right\} \cup\{0\}
\end{aligned}
$$

Заметим далее, что энтропийное условие (16) справедливо и для лишь непрерывных сублинейных энтропий $h(u)$. Последнее легко доказывается с помощью равномерной на любом компакте аппроксимации функции $h(u)$ последовательностью сублинейных функций $h_{k}(u) \in C^{1}\left(\mathbb{R}^{n} \backslash\{0\}\right), k \in \mathbb{N}$, и последующего предельного перехода при $k \rightarrow \infty$ в условии $(16): h_{k}(u)_{t}+\left(\varphi(|u|) h_{k}(u)\right)_{x} \leqslant 0$ в $\mathscr{D}^{\prime}(\Pi)$.

Положим $h(u)=\left(\delta|u|-u_{1}\right)^{+}=\max \left(\delta|u|-u_{1}, 0\right)$. Тогда непрерывная функция $h(u)$ сублинейна как максимум сублинейных функций, неотрицательна и $h(u)=0$ в точности на множестве $\Gamma(\delta)$. В частности, $h\left(u_{0}\right)=0$ п.в. на $\mathbb{R}$.

Если $u=u(t, x)$ - о.э.р. задачи $(1),(2)$ с начальными данными $u_{0}=u_{0}(x)$, то в силу принципа максимума из следствия $1|u(t, x)| \leqslant M=\operatorname{ess} \sup \left|u_{0}(x)\right|$ п.в. на П. 
Пусть $\omega(\sigma)=\sup \{|f(y)-f(z)|: y, z \in[0, M],|y-z| \leqslant \sigma\}-$ модуль непрерывности функции $f(u)$ на отрезке $[0, M]$. Ясно, что $\omega(\sigma)-$ неубывающая субаддитивная функция на $\mathbb{R}_{+}, \lim _{\sigma \rightarrow 0+} \omega(\sigma)=0$. Так как $f(0)=0$, то $|f(r)|=|f(r)-f(0)| \leqslant \omega(r)$ при $r \in[0, M]$.

По лемме $3 \forall \varepsilon>0|f(r)| \leqslant \omega(r) \leqslant(r+\varepsilon) \omega(\varepsilon) / \varepsilon$ и для потока $q(u)=\varphi(r) h(u)=$ $f(r) h(u / r), r=|u|$, энтропии $h(u)$ справедлива оценка: $\forall \varepsilon>0$

$$
|q(u)| \leqslant \frac{\omega(\varepsilon)}{\varepsilon}(r+\varepsilon) h\left(\frac{u}{r}\right)=\frac{\omega(\varepsilon)}{\varepsilon}\left(r h\left(\frac{u}{r}\right)+\varepsilon h\left(\frac{u}{r}\right)\right) \leqslant \frac{\omega(\varepsilon)}{\varepsilon}(h(u)+\varepsilon)
$$

(здесь использовано также, что $h(u / r) \leqslant 1$ ).

Пусть $N(\varepsilon)=\omega(\varepsilon) / \varepsilon+1$. Тогда $N(\varepsilon) \geqslant 1$ и для почти всех $(t, x) \in \Pi$

$$
|q(u(t, x))| \leqslant N(\varepsilon)(h(u(t, x))+\varepsilon) .
$$

По условиям (16), (17) имеем также $h(u)_{t}+q(u)_{x} \leqslant 0$ в $\mathscr{D}^{\prime}(\Pi)$ и $\underset{t \rightarrow 0+}{\operatorname{ess}} \lim _{t \rightarrow 0} h(u(t, x))=$ $h\left(u_{0}(x)\right)=0$ в $L_{\mathrm{loc}}^{1}(\mathbb{R})$. Таким образом, функции $\alpha(t, x)=h(u(t, x)), \beta(t, x)=$ $q(u(t, x))$ удовлетворяют условиям леммы 4 , и по этой лемме для почти всех $t>0$

$$
\int h(u(t, x)) e^{-|x|} d x \leqslant e^{t} \cdot \inf _{\varepsilon>0} 2 \varepsilon N(\varepsilon)=0,
$$

так как $\varepsilon N(\varepsilon)=\varepsilon+\omega(\varepsilon) \rightarrow 0$ при $\varepsilon \rightarrow 0+$. Отсюда следует, что $h(u(t, x))=0$ п.в. на П. С учетом свойств функции $h(u)$ это эквивалентно включению $u(t, x) \in \Gamma(\delta)$ п.в. на П, которое и нужно было установить.

Переходя к доказательству утверждения теоремы, касающегося множества $\dot{\Gamma}(\delta)$, предположим, что $u_{0}(x) \in \dot{\Gamma}(\delta)$ п.в. на $\mathbb{R}$. Тогда, как это уже доказано выше, $u(t, x) \in \Gamma(\delta)$ п.в. на П.

Рассмотрим далее последовательность непрерывных сублинейных функций $h_{k}(u)=\left(|u|+k\left(\delta|u|-u_{1}\right)\right)^{+}, k \in \mathbb{N}$. Ясно, что при $u \in \Gamma(\delta)\left(\right.$ когда $\left.\delta|u|-u_{1} \leqslant 0\right)$ $0 \leqslant h_{k}(u) \leqslant|u|$ и

$$
h_{k}(u) \underset{k \rightarrow \infty}{\longrightarrow} h(u)= \begin{cases}0, & \delta|u|-u_{1}<0 \\ |u|, & \delta|u|-u_{1}=0\end{cases}
$$

Легко видеть, что $h(u)=0$ тогда и только тогда, когда $u \in \dot{\Gamma}(\delta)$.

Повторяя рассуждения из первой части доказательства и применяя лемму 4 при $\alpha(t, x)=h_{k}(u(t, x)), \beta(t, x)=q_{k}(u(t, x))$ (заметим, что $u=u(t, x) \in \Gamma(\delta)$ п.в. на П и, значит, $h_{k}(u / r) \leqslant 1$, откуда вытекает справедливость оценки (26) с $h(u)=h_{k}(u), q=q_{k}(u)=\varphi(r) h_{k}(u), r=|u|$, для всех натуральных $\left.k\right)$, получаем, что при любом $\varepsilon>0$ для почти всех $t>0$

$$
\int h_{k}(u(t, x)) e^{-|x|} d x \leqslant e^{t} \cdot\left(\int h_{k}\left(u_{0}(x)\right) e^{-|x| / N(\varepsilon)} d x+2 \varepsilon N(\varepsilon)\right) .
$$

Далее, $u_{0}(x) \in \dot{\Gamma}(\delta)$ п.в. на $\mathbb{R}, u(t, x) \in \Gamma(\delta)$ п.в. на П. Тогда ввиду $(27)$ при $k \rightarrow \infty$

$$
h_{k}\left(u_{0}\right) \rightarrow h\left(u_{0}\right)=0 \text { п.в. на } \mathbb{R}, \quad h_{k}(u) \rightarrow h(u) \text { п.в. на } \Pi,
$$


и по теореме Лебега об ограниченной сходимости в пределе при $k \rightarrow \infty$ из (28) следует оценка: для почти всех $t>0$

$$
\int h(u(t, x)) e^{-|x|} d x \leqslant 2 e^{t} \varepsilon N(\varepsilon)
$$

из которой ввиду произвольности $\varepsilon>0$ вытекает, что

$$
\int h(u(t, x)) e^{-|x|} d x=0 \text { для почти всех } t>0 .
$$

Это означает, что $h(u)=0$ п.в. на П, т.е. для почти всех значений аргументов $u(t, x) \in \dot{\Gamma}(\delta)$, что и требовалось доказать.

СЛЕДСТВИЕ 2. Пусть $h\left(u_{0}\right)=0$ n.в. на $\mathbb{R}$, әде $h(u)=(c, u)-$ линейная функция, $u=u(t, x)$ - о.э.р. задачи (1), (2). Тогда $h(u)=0$ п.в. на П.

Для доказательства нужно лишь заметить, что гиперплоскость $h(u)=0$ есть $\Gamma(e, 0) \cap \Gamma(-e, 0), e=c /|c|$.

\section{§ 3. Сильные о.э.p.}

Несмотря на ограничительное энтропийное условие (16) о.э.р. задачи (1), (2) может быть неединственно (см. пример ниже). В связи с этим мы вводим понятие сильного о.э.р. Как будет установлено ниже, сильные о.э.р. образуют класс сушествования и единственности для рассматриваемой задачи.

ОПРЕДЕЛЕНИЕ 5. Ограниченная измеримая вектор-функция $u=u(t, x)$ называется сильным о.э.р., если функция $r=|u(t, x)|$ является о.э.р. скалярной задачи $(23),(24)$ с начальными данньми $r_{0}=\left|u_{0}\right| ; u_{t}+(\varphi(r) u)_{x}=0$ в $\mathscr{D}^{\prime}\left(\Pi, \mathbb{R}^{n}\right)$ и выполнено начальное условие (17).

Если существует классическое решение $u=u(t, x)$ задачи $(1),(2)$, то оно является также и сильным о.э.р. Действительно, предположим для простоты, что $f(r) \in C^{1}$. Тогда из соотношения (10) при $p(u)=\eta(|u|)$ следует, что функция $r=|u(t, x)|$ удовлетворяет условию а) определения 3 (причем со знаком равенства). Поскольку условие b) также выполнено, то $r$ - о.э.р. задачи (23), (24) с начальными данными $r_{0}=\left|u_{0}\right|$. Таким образом, $u$ удовлетворяет требованиям определения 5 .

Если $u=u(t, x)$ - сильное о.э.р. задачи (1), (2), то по определению 5 функция $r=|u(t, x)|-$ единственное о.э.р. задачи $(23),(24)$ с начальной функцией $r_{0}=$ $\left|u_{0}(x)\right|$, а неизвестные $v_{i}=u_{i} / r, i=1, \ldots, n$ (при $r=0$ значения этих функций не играют роли), должны удовлетворять в $\mathscr{D}^{\prime}(\Pi)$ линейному уравнению

$$
\begin{gathered}
(A v)_{t}+(B v)_{x}=0, \\
A=A(t, x)=r(t, x), \quad B=B(t, x)=f(r(t, x)),
\end{gathered}
$$

с начальным условием

$$
v(0, x)=v_{0}(x)
$$

в смысле следуюшего определения $\left(\right.$ с $\left.A(0, x)=r_{0}(x)\right)$ : 
ОПРЕДЕЛЕНИЕ 6 (см. [1], [2]). Функция $v=v(t, x) \in L^{\infty}(\Pi)$ называется $о 6$ общенным решением (коротко - о.р.) задачи Коши (29), (30), если для любой пробной функции $h=h(t, x)$ из пространства $C_{0}^{\infty}(\bar{\Pi})$ бесконечно дифференцируемых функций с компактным носителем в $\bar{\Pi}=[0,+\infty) \times \mathbb{R}$

$$
\int_{\Pi}\left[A v h_{t}+B v h_{x}\right] d t d x+\int_{\mathbb{R}} A(0, x) v_{0}(x) h(0, x) d x=0 .
$$

Выбирая в (31) пробные функции $h \in C_{0}^{\infty}(\Pi)$, получаем, что $(A v)_{t}+(B v)_{x}=0$ в $\mathscr{D}^{\prime}(\Pi)$. Тождество (31) учитьвает также начальное условие $(30)$. Точнее, $(31)$ эквивалентно равенству $(A v)_{t}+(B v)_{x}=0$ в $\mathscr{D}^{\prime}(\Pi)$ и начальному условию $(30)$, понимаемому в смысле $*$-слабой сходимости $A(t, \cdot) v(t, \cdot) \rightarrow A(0, \cdot) v_{0}$ в $L^{\infty}(\mathbb{R})$, когда $t \rightarrow 0+$, пробегая некоторое множество полной меры.

В работе [2] исследована разрешимость задачи $(29),(30)$ и установлены свойства о.p. в случае произвольных коэффициентов $A, B \in L^{\infty}(\Pi)$ со свойствами:

$$
\begin{gathered}
\underset{t \rightarrow 0+}{\operatorname{ess} \lim _{t \rightarrow 0}} A(t, x)=A(0, x) \text { в } L_{\text {lос }}^{1}(\mathbb{R}), \quad A(0, x) \in L^{\infty}(\mathbb{R}) ; \\
\forall \varepsilon>0|B| \leqslant N(\varepsilon) \cdot(A+\varepsilon) \text { п.в. на } \Pi, \varepsilon N(\varepsilon) \underset{\varepsilon \rightarrow 0+}{\longrightarrow} 0 ; \\
A_{t}+B_{x}=0 \text { в } \mathscr{D}^{\prime}(\Pi) .
\end{gathered}
$$

Прежде всего установим, что коэффициенты $A=r(t, x), B=f(r(t, x))$ удовлетворяют условиям (32)-(34). Ясно, что $A(0, x) \geqslant 0, A(t, x) \geqslant 0$. По условию b) определения 3 имеем также

$$
\underset{t \rightarrow 0+}{\operatorname{ess} \lim _{t \rightarrow 0}} A(t, x)=A(0, x) \quad \text { в } \quad L_{\text {loc }}^{1}(\mathbb{R})
$$

таким образом, выполнено соотношение (32). Далее, пусть $\omega(\sigma)$ - модуль непрерывности функции $f(r)$ на отрезке $[0, M], M=\operatorname{ess} \sup |u(t, x)|$. Тогда $r=r(t, x) \in$ $[0, M]$ п.в. на П и по лемме $3 \forall \varepsilon>0$

$$
|B(t, x)|=|f(r)|=|f(r)-f(0)| \leqslant \omega(r) \leqslant \frac{\omega(\varepsilon)}{\varepsilon}(r+\varepsilon)=\frac{\omega(\varepsilon)}{\varepsilon}(A(t, x)+\varepsilon),
$$

т.е. верна оценка $(33)$ с $N(\varepsilon)=\omega(\varepsilon) / \varepsilon$. Наконец, поскольку $r=r(t, x)$ - о.э.р. задачи $(23),(24)$, то в $\mathscr{D}^{\prime}(\Pi)$

$$
A_{t}+B_{x}=r_{t}+f(r)_{x}=0
$$

и выполнено условие (34).

Заметим, что из условия (33) легко следует, что $A(t, x) \geqslant 0$ и $B(t, x)=0$ при $A(t, x)=0$ п.в. на П. В частности, как видно из определения 6 , значение о.p. $v(t, x)$ при $A(t, x)=0$ не играет роли. В этом смысле можно рассматривать задачу $(29),(30)$ как задачу Коши относительно неизвестной функции $A v$ с начальными данными $A(0, x) v_{0}(x)$.

В [2] доказаны следующие утверждения. 
ПРЕДЛОЖЕНИЕ 4. 1) Существует о.p. v(t,x) задачи (29), (30);

2) $\operatorname{ess} \lim _{t \rightarrow 0+} A(t, x) v(t, x)=A(0, x) v_{0}(x)$ в $L_{\text {loc }}^{1}(\mathbb{R})$;

3) $\forall p(u) \in C^{1}(\mathbb{R})$ функция $p(v(t, x))$ является о.р. задачи (29), (30) с начальными даннымми $p\left(v_{0}(x)\right)$;

4) если $v_{0}(x) A(0, x)=0$ n.в. на $\mathbb{R}$, то $v(t, x) A(t, x)=0$ п.в. на П (единственность).

Из утверждения 3) следует, что множество о.р. образует алгебру. Действительно, если $v_{1}, v_{2}-$ о.р. задачи (29), (30) (со своими начальными данными), то таковьм будет и $v_{1} v_{2}=\left(\left(v_{1}+v_{2}\right)^{2}-v_{1}^{2}-v_{2}^{2}\right) / 2$. В частности, если $v_{i}=v_{i}(t, x)$, $i=1, \ldots, m,-$ конечное множество о.р. задачи $(29),(30)$ с начальными данньми $v_{0 i}, p(z)$-многочлен от переменных $z=\left(z_{1}, \ldots, z_{m}\right)$, то функция $v=p\left(v_{1}, \ldots, v_{m}\right)$ также является о.р. задачи (29), (30) с начальными данными $v_{0}=p\left(v_{01}, \ldots, v_{0 m}\right)$.

Заметим далее, что определение 6 имеет смысл и для неограниченных функций $v(t, x), v_{0}(x)$ таких, что $A(0, x) v_{0}(x) \in L^{\infty}(\mathbb{R}), A v, B v \in L^{\infty}(\Pi)$. В этом общем случае в [2] доказано

ПРЕДЛОЖЕНИЕ 5. Пусть $v=v(t, x)$ - о.р. задачи (29), (30) с начальной функиией $v_{0}(x)$. Тогда

1) о.р. v единственно: если $v_{0}(x) A(0, x)=0$ п.в. на $\mathbb{R}$, mo $v(t, x) A(t, x)=0$ п.в. на $\Pi$;

2) если $p(v) \in C^{1}(\mathbb{R}),|p(v)| \leqslant \mathrm{const} \cdot(|v|+1)$, то функиия $p(v(t, x))$ является о.р. задачи (29), (30) с начальнымми данными $p\left(v_{0}(x)\right)$.

Нам потребуется следующее обобщение утверждения 2).

ПРЕДЛОЖЕНИЕ 6. Если $v_{i}=v_{i}(t, x), i=1, \ldots, m,-$ o.p. задачи (29), (30) $c$ начальными данными $v_{0 i}, p(v) \in C\left(\mathbb{R}^{m}\right)$ и для почти всех $(t, x)$

$$
|p(v)| \leqslant c(|v|+1), \quad v=\left(v_{1}(t, x), \ldots, v_{m}(t, x)\right), \quad c=\mathrm{const},
$$

то функиия $p\left(v_{1}, \ldots, v_{m}\right)$ является о.р. задачи $(29),(30)$ с начальными данными $p\left(v_{01}, \ldots, v_{0 m}\right)$.

ДокАЗАТЕЛьство. Предположим сначала, что $v_{0 i} \in L^{\infty}(\mathbb{R}), v_{i} \in L^{\infty}(\Pi)$, $i=1, \ldots, m$. Тогда условие $(35)$ выполнено для всех непрерывных $p(z)$. Если $p(z)$ - многочлен, то предложение верно ввиду того, что множество ограниченных о.р. задачи (29), (30) образует алгебру. Аппроксимируя произвольную непрерывную функцию равномерно на любом компакте в $\mathbb{R}^{m}$ последовательностью многочленов и переходя к пределу в соответствуюшей последовательности соотношений (31), получаем справедливость предложения для всех $p(z) \in C\left(\mathbb{R}^{m}\right)$.

В обшем случае рассмотрим последовательность ограниченных гладких функций $s_{k}(u)$ на $\mathbb{R}$ со свойствами: $\left|s_{k}(u)\right| \leqslant|u|, s_{k}(u) \rightarrow u$ при $k \rightarrow \infty \forall u \in \mathbb{R}$ (например, можно положить $s_{k}(u)=e^{-|u| / k} u$ ).

По утверждению 2) предложения 5 функции $v_{i}^{k}=s_{k}\left(v_{i}\right)$ являются ограниченными o.p. задачи $(29),(30)$ с начальными данными $v_{0 i}^{k}=s_{k}\left(v_{0 i}\right) \in L^{\infty}(\mathbb{R})$ и, как уже установлено выше, $p^{k}=p\left(v_{1}^{k}, \ldots, v_{m}^{k}\right)$ - o.p. задачи $(29),(30)$ с начальньпи данными $p_{0}^{k}=p\left(v_{01}^{k}, \ldots, v_{0 m}^{k}\right)$, т.е. $\forall h=h(t, x) \in C_{0}^{\infty}(\bar{\Pi})$

$$
\int_{\Pi}\left[A p^{k} h_{t}+B p^{k} h_{x}\right] d t d x+\int_{\mathbb{R}} A(0, x) p_{0}^{k}(x) h(0, x) d x=0 .
$$


По построению при $k \rightarrow \infty$

$$
\begin{aligned}
p^{k}(t, x) \rightarrow p(t, x) & =p\left(v_{1}(t, x), \ldots, v_{m}(t, x)\right), \\
p_{0}^{k}(x) \rightarrow p_{0}(x) & =p\left(v_{01}(x), \ldots, v_{0 m}(x)\right)
\end{aligned}
$$

и ввиду условий $\left|s_{k}(u)\right| \leqslant|u|$ и $(35)$ при $v=\left(v_{1}, \ldots, v_{m}\right), v_{0}=\left(v_{01}, \ldots, v_{0 m}\right)$ справедливы оценки

$$
\left|A p^{k}\right| \leqslant c A(1+|v|), \quad\left|A(0, x) p_{0}^{k}\right| \leqslant c A(0, x)\left(1+\left|v_{0}\right|\right), \quad\left|B p^{k}\right| \leqslant c|B|(1+|v|),
$$

правые части которых лежат в пространствах $L^{\infty}$ (поскольку $A(0, x) v_{0 i}(x) \in$ $\left.L^{\infty}(\mathbb{R}), A v_{i}, B v_{i} \in L^{\infty}(\Pi)\right)$. Это позволяет применить теорему Лебега при предельном переходе под знаками интегралов в (36) и вывести в пределе при $k \rightarrow \infty$, что $\forall h=h(t, x) \in C_{0}^{\infty}(\bar{\Pi})$

$$
\int_{\Pi}\left[A p h_{t}+B p h_{x}\right] d t d x+\int_{\mathbb{R}} A(0, x) p_{0}(x) h(0, x) d x=0 .
$$

Таким образом, функция $p=p\left(v_{1}, \ldots, v_{m}\right)$ является о.р. задачи $(29),(30)$ с начальными данными $p_{0}=p\left(v_{01}, \ldots, v_{0 m}\right)$.

Возвратимся к исследованию задачи (1), (2). Верна следующая

Tеорема 5. Пусть $A=r, B=f(r) ; v_{i}=v_{i}(t, x)$ - o.p. задачи (29), (30) (существующие по предложению 4) с начальными данными $v_{0 i}=u_{0 i} / r_{0}, i=$ $1, \ldots, n\left(\right.$ при $r_{0}=0$ можно положить $\left.v_{0 i}=0\right)$. Тогда вектор-функиия $u=r v$, $v=\left(v_{1}, \ldots, v_{n}\right)$, является единственным сильным о.э.р. задачи (1), (2).

ДокАЗАТЕЛЬСТво. Поскольку $\|r\|_{\infty}=M<\infty$, то $u=r v \in L^{\infty}\left(\Pi, \mathbb{R}^{n}\right)$. По условию вектор-функция $v(t, x)$ удовлетворяет уравнению $(29)$ и, значит,

$$
u_{t}+(\varphi(r) u)_{x}=(r v)_{t}+(f(r) v)_{x}=0 \quad \text { в } \mathscr{D}^{\prime}(\Pi) .
$$

Напомним, что $r=r(t, x)$ - о.э.р. задачи $(23),(24)$ с начальной функцией $r_{0}=$ $\left|u_{0}(x)\right|$. С учетом свойства 3) из предложения 4 (или предложения 6) функция $|v|^{2}=v_{1}^{2}+\cdots+v_{n}^{2}$ является о.р. задачи (29), (30) с начальной функцией $\left|v_{0}\right|^{2}$. Так как $r_{0}\left|v_{0}\right|^{2} \equiv r_{0} \cdot 1$, то о.р. этой задачи является также постоянная, равная единице. В силу единственности (см. свойство 4) в предложении 4) $r|v|^{2}=r$ п.в. на П. Умножив это равенство на $r$, получим, что $|u|=r$ п.в. на П и, значит, $|u|-$ о.э.р. задачи $(23),(24)$ с начальной функцией $r_{0}=\left|u_{0}(x)\right|$. Кроме того, поскольку в $(37) r=|u|$, то $u$ удовлетворяет системе (1) в смысле распределений. Наконец, как следует из свойства 2) предложения 4 , для $u$ вьполнено начальное условие (17). Итак, все требования определения 5 выполнены и $u=u(t, x)$ - сильное о.э.р. задачи $(1),(2)$.

Единственность этого решения вытекает из того, что, во-первых, функция $r=$ $r(t, x)$ однозначно определяется как о.э.р. задачи $(23),(24)$ с начальной функцией $r_{0}=\left|u_{0}(x)\right|$. Во-вторых, тогда вектор-функция $u=r v$ однозначно (по свойству 4 ) предложения 4) определяется тем условием, что $v=v(t, x)-$ o.p. задачи $(29),(30)$ (при $A=r, B=f(r))$ с $r_{0} v_{0}=u_{0}$. Теорема доказана. 
ТЕОРема 6. Сильное о.э.р. задачи (1), (2) является и о.э.р. этой задачи.

ДокАЗАТЕЛЬСТво. По предложению 1 любая допустимая вьпуклая энтропия имеет вид $p(u)=\eta(|u|)+h(u)$ с выпуклой на $\mathbb{R}_{+}$функцией $\eta(r)$ и однородной функцией $h(u)$; соответствующий поток есть $q(u)=\psi(|u|)+\varphi(|u|) h(u)$, где функция $\psi(r)$ определена равенством (22). Если $u=u(t, x)$ - сильное о.э.р. задачи $(1),(2)$, то по определению $5 r=|u|-$ о.э.р. скалярной задачи (23), (24), и в силу условия а) определения 3

$$
\eta(|u|)_{t}+\psi(|u|)_{x} \leqslant 0 \quad \text { в } \mathscr{D}^{\prime}(\Pi)
$$

По теореме 5 компоненты вектор-функции $v=u / r$ являются о.р. задачи $(29),(30)$ при $A=r, B=f(r)$. По предложению 6 функция $h(u / r)$ - также о.р. этой задачи (с соответствующими начальньми данными) и, в частности, $(r h(u / r))_{t}+$ $(f(r) h(u / r))_{x}=0$ в $\mathscr{D}^{\prime}(\Pi)$. Ввиду однородности $r h(u / r)=h(u)$ и последнее равенство переписывается как

$$
h(u)_{t}+(\varphi(|u|) h(u))_{x}=0 \quad \text { в } \mathscr{D}^{\prime}(\Pi) .
$$

Складывая (38) и (39), получаем соотношение

$$
p(u)_{t}+q(u)_{x} \leqslant 0 \text { в } \mathscr{D}^{\prime}(\Pi)
$$

справедливое для любой допустимой выпуклой энтропии $p(u)$ и соответствующего потока $q(u)$. По определению $2 u=u(t, x)$ - о.э.р. задачи (1), (2) (заметим, что начальное условие (17) содержится в определении сильного о.э.р.). Теорема доказана.

Из теорем 5, 6, в частности, следует существование о.э.р. задачи (1), (2). Покажем, что в случае, когда начальная вектор-функция $u_{0}(x)$ удовлетворяет условию

(C) для некоторого вектора $e \in \mathbb{R}^{n}$ единичной длины $u_{0}(x) \in \dot{\Gamma}(e, 0)$ п.в. на $\mathbb{R}$, верна также единственность о.э.р. Для этого нам понадобится следующая простая

Лемма 5. Пусть $r=r(t, x)$ - о.э.суб-р. (или о.э.супер-р.) скалярной задачи Коши (23), (24), удовлетворяющее уравнению (23) в смысле распределений и строгому начальному условию (в смысле требования b) определения 3 ). Тогда и $(t, x)$ - о.э.р. задачи (23), (24).

Для доказательства нужно лишь заметить, что произвольная выпуклая функция $\eta(r)$ допускает представление $\eta(r)=C r+\eta(r)-C r$, где при достаточно большом $C>0$ функция $C r+\eta(r)$ выпукла и не убьвает на $[-M, M], M=\|r\|_{\infty}$. Подробности доказательства леммы 5 содержатся, например, в [2].

ТЕОРЕМА 7. При выполнении условия (C) о.ә.р. и $(t, x)$ задачи $(1),(2)$ является сильнымм и, тем самым, единственным. 
ДокАЗАТЕльство. По замечанию 2 мы можем применить ортогональное преобразование вектора $u=u(t, x)$ и свести доказательство к случаю, когда $e=$ $(1,0, \ldots, 0)$ - первый базисный вектор. В этом случае условие $(\mathrm{C})$ означает, что $u_{01}>0$ или $r_{0}=\left|u_{0}\right|=0$ п.в. на $\mathbb{R}$. Рассмотрим задачу (29), (30) с коэффициентами $A=A(t, x)=u_{1}(t, x), B=B(t, x)=\varphi(r) u_{1}(t, x), r=|u|$. Положим $A(0, x)=u_{01}(x)$. Заметим, что коэффициенты $A, B$ удовлетворяют условиям (32)-(34). Действительно, (32) следует из начального требования (17) определения 2. Условие (34) вытекает из того, что $u$ удовлетворяет системе (1) в смысле распределений (см. замечание 1$)$. Далее, пусть $\omega(\sigma)$-модуль непрерывности функции $f(r)$ на отрезке $[0, M], M=\operatorname{ess} \sup |u(t, x)|$. Тогда $r=|u(t, x)| \in[0, M]$ п.в. на П и по лемме $3 \forall \varepsilon>0$

$$
|B(t, x)|=\left|f(r) \frac{u_{1}}{r}\right| \leqslant \omega(r) \frac{u_{1}}{r} \leqslant \frac{\omega(\varepsilon)}{\varepsilon}(r+\varepsilon) \frac{u_{1}}{r} \leqslant \frac{\omega(\varepsilon)}{\varepsilon}(A(t, x)+\varepsilon)
$$

(здесь при $r=0$ значение $u_{1} / r$ можно выбрать произвольно), т.е. верна оценка (33) c $N(\varepsilon)=\omega(\varepsilon) / \varepsilon$.

По теореме $4 u(t, x) \in \dot{\Gamma}(e, 0)$ п.в. на П, т.е. $u_{1}>0$ или $r=0$. Положим

$$
v_{0 i}=v_{0 i}(x)=\left\{\begin{array}{ll}
\frac{u_{0 i}}{u_{01}}, & u_{01}>0, \\
0, & u_{01} \leqslant 0,
\end{array} \quad v_{i}=v_{i}(t, x)= \begin{cases}\frac{u_{i}}{u_{1}}, & u_{1}>0 \\
0, & u_{1} \leqslant 0\end{cases}\right.
$$

$i=1, \ldots, n$. Заметим, что $A v_{i}=u_{i}, B v_{i}=\varphi(r) u_{i}$ п.в. на П. Действительно, это очевидно при $u_{1}>0$ и при $r=0$ (тогда $u_{i}=v_{i}=0$ ), а по крайней мере одно из этих условий выполнено п.в. на П. Аналогично устанавливается, что $A(0, x) v_{0 i}=u_{0 i}$. С учетом замечания 1 имеем тогда

$$
\left(A v_{i}\right)_{t}+\left(B v_{i}\right)_{x}=\left(u_{i}\right)_{t}+\left(\varphi(r) u_{i}\right)_{x}=0 \quad \text { в } \mathscr{D}^{\prime}(\Pi)
$$

Кроме того, как следует из требования (17),

$$
\underset{t \rightarrow 0+}{\operatorname{ess} \lim _{t \rightarrow 0}} A(t, x) v_{i}(t, x)=\underset{t \rightarrow 0+}{\operatorname{ess} \lim _{i}} u_{i}(t, x)=u_{0 i}(x)=A(0, x) v_{0 i}(x) \quad \text { в } \quad L_{\mathrm{loc}}^{1}(\mathbb{R}) .
$$

Из соотношений, приведенных выше, легко следует (см., например, [2; предложение 7]), что при $v=v_{i}$ выполнено определяюшее условие $(31)$ и $v_{i}$ - (возможно неограниченное) о.р. задачи (29), (30) с начальной функцией $v_{0 i}$, причем $A v_{i}, B v_{i} \in$ $L^{\infty}(\Pi), A(0, x) v_{0 i}(x) \in L^{\infty}(\mathbb{R})$. Положим $v=\left(v_{1}, \ldots, v_{n}\right), v_{0}=\left(v_{01}, \ldots, v_{0 n}\right)$. По предложению 6 функция $w=|v|$ является о.р. задачи (29), (30) с начальными данными $w_{0}=\left|v_{0}\right|$ и, значит, $r_{t}+f(r)_{x}=(A w)_{t}+(B w)_{x}=0$ в $\mathscr{D}^{\prime}(\Pi)$, т.е. функция $r=r(t, x)$ удовлетворяет уравнению (23) в смысле распределений. По теореме 3 $r$ является также о.э.суб-р. задачи (23), (24) со строгим начальным условием. Поэтому в силу леммы $5 r=|u|$ - о.э.р. задачи (23), (24), и выполнены требования определения 5. Итак, $u$ - сильное о.э.р., единственное по теореме 5. Теорема доказана.

Как иллюстрируется примером ниже, в условии $(\mathrm{C})$ теоремы 7 нельзя заменить конус $\dot{\Gamma}(e, 0)$ на $\Gamma(e, 0)$. 
ПримеР. Рассмотрим случай $n=2, \varphi(r)=r$. Тогда система (1) имеет вид

$$
u_{t}+(r u)_{x}=0, \quad u=\left(u_{1}, u_{2}\right), \quad r=|u|=\left(u_{1}^{2}+u_{2}^{2}\right)^{1 / 2} .
$$

Поставим следующие начальные условия

$$
u(0, x)=u_{0}(x)=(0, \operatorname{sign} x) .
$$

Заметим, что здесь $u_{0} \in \Gamma(e, 0)$ при $e=(1,0)$. Поскольку $r_{0}=\left|u_{0}\right| \equiv 1$, то для сильного о.э.p. $u=u(t, x)$ задачи (40), (41) $r=|u| \equiv 1$ (как о.э.р. соответствующей скалярной задачи с начальной функцией $\left.r_{0} \equiv 1\right)$ и $u_{t}+u_{x}=0$ в $\mathscr{D}^{\prime}\left(\Pi, \mathbb{R}^{2}\right)$. Поэтому $u(t, x)=u_{0}(x-t)=(0, \operatorname{sign}(x-t))$ - единственное сильное о.э.р. задачи (40), (41).

Пусть далее $v=v(t, x)$ - о.э.р. задачи Коши для скалярного уравнения

$$
v_{t}+(|v| v)_{x}=0
$$

с начальной функцией $\operatorname{sign} x$. Легко проверить, что

$$
v(t, x)= \begin{cases}-1, & x<2(\sqrt{2}-1) t \\ \frac{x}{2 t}, & 2(\sqrt{2}-1) t \leqslant x \leqslant 2 t, \\ 1, & x>2 t\end{cases}
$$

и, как показано в работе [5], решение $v(t, x)$ является пределом в $L_{\text {loc }}^{1}(\Pi)$ семейства классических решений $v^{\varepsilon}(t, x) \in C^{2}(\Pi)$ задачи Коши для уравнения $v_{t}+(|v| v)_{x}=$ $\varepsilon v_{x x}$ при стремлении "вязкости" $\varepsilon$ к нулю. Тогда при $\bar{u}(t, x)=(0, v(t, x)), u^{\varepsilon}(t, x)=$ $\left(0, v^{\varepsilon}(t, x)\right)$ вектор-функция $u^{\varepsilon}$ является $C^{2}$-гладким решением параболической системы $(18)$ и $u^{\varepsilon}(t, x) \rightarrow \bar{u}(t, x)$ в $L_{\text {loc }}^{1}\left(\Pi, \mathbb{R}^{2}\right)$ при $\varepsilon \rightarrow 0$. В соответствии с предложением $2 \bar{u}(t, x)$ удовлетворяет энтропийному условию (16) для любой выпуклой энтропии $p(u) \in \mathscr{F}$, и поскольку справедливость начального условия (17) очевидна, то $\bar{u}(t, x)$ также является о.э.р. задачи $(40),(41)$.

Итак, $u(t, x), \bar{u}(t, x)$ - два различных о.э.р. задачи $(40),(41)$.

ЗАмечАниЕ 3 . Если $u=u(t, x)$ - сильное о.э.р. задачи $(1),(2), r=|u|$, то $X$-значная функция $(X-$ пространство симметричных $(n \times n)$-матриц $) U=$ $U(t, x)=u \otimes u / r$ с компонентами $U_{i j}=\left\{\begin{array}{ll}u_{i} u_{j} / r, & r>0, \\ 0, & r=0,\end{array}\right.$ является единственньм сильным о.э.р. для системы $(4)$ при $f(u)=\varphi(|u|) u$ с соответствующим начальным условием

$$
U(0, x)=U_{0}(x)=u_{0} \otimes u_{0} / r_{0}, \quad r_{0}=\left|u_{0}\right|,
$$

т.е. (см. [1], [2]) выполнены условия:

(C1) собственные числа $\lambda_{1} \leqslant \cdots \leqslant \lambda_{n}$ матрищы $U$ являются о.э.р. задачи (23), (24) с начальньми данньми $\lambda_{01} \leqslant \cdots \leqslant \lambda_{0 n}-$ собственными числами матрицы $U_{0}$;

(C2) $U_{t}+\tilde{f}(U)_{x}=0$ в $\mathscr{D}^{\prime}(\Pi, X)$;

(C3) $\operatorname{ess} \lim _{t \rightarrow 0+} U(t, \cdot)=U_{0}$ в $L_{\text {loc }}^{1}(\mathbb{R}, X)$. 
Действительно, здесь $\lambda_{1}=\cdots=\lambda_{n-1}=0, \lambda_{01}=\cdots=\lambda_{0 n-1}=0, \lambda_{n}=r(t, x)$, $\lambda_{0 n}=r_{0}(x)$, и так как по определению $5 r(t, x)$ - о.э.р. задачи $(23),(24)$ с начальньми данньми $r_{0}$, то требование (C1) вьполнено. Далее, при $U=u \otimes u / r$ верно равенство $\tilde{f}(U)=\varphi(r) U$, и система (4) переписьвается в виде $U_{t}+\varphi(r) U=0$. По теореме 5 при $i=1, \ldots, n$ функции $v_{i}=u_{i} / r$ (полагаем $v_{i}=0$ при $r=0$ ) являются ограниченньпи о.р. линейной задачи (29), (30). По предложению 6 тогда $v_{i} v_{j}$, $i, j=1, \ldots, n$, также будут о.р. этой задачи. Поэтому

$$
\left(U_{i j}\right)_{t}+\left(\varphi(r) U_{i j}\right)_{x}=\left(r v_{i} v_{j}\right)_{t}+\left(f(r) v_{i} v_{j}\right)_{x}=0 \quad \text { в } \quad \mathscr{D}^{\prime}(\Pi)
$$

и требование (C2) также выполнено. Справедливость (C3) непосредственно следует из начального условия $(17)$ для $u(t, x)$.

Итак, $U(t, x)$ - сильное о.э.р. задачи $(4),(42)$.

Докажем единственность сильного о.э.р. $U(t, x)$. Как следует из $(\mathrm{C} 1)$, собственные числа $\lambda_{1}=\cdots=\lambda_{n-1}=0$ как о.э.р. задачи $(23),(24)$ с нулевыми начальными данными $\lambda_{01}=\cdots=\lambda_{0 n-1}$, а $\lambda_{n} \geqslant 0$ п.в. на П, что вытекает по принципу сравнения из неотрицательности $\lambda_{0 n}=r_{0}(x)$ (см. предложение 3 ). Таким образом, матрица $U$ неотрицательно определена и имеет ранг не больший единицы. Поэтому найдется представление $U=u \otimes u /|u|$ с некоторым вектором $u=u(t, x) \in L^{\infty}\left(\Pi, \mathbb{R}^{n}\right)$, при этом $r=|u|=\lambda_{n}$ - единственное о.э.р. задачи $(23),(24)$ с начальной функцией $r_{0}$. Ввиду условий (C2), (С3) при $i, j=1, \ldots, n$ функции $v=v_{i j}=u_{i} u_{j} / r^{2}$ (значения которых полагаем равньми нулю при $r=0)$ - о.р. линейной задачи $(29),(30):(r v)_{t}+(f(r) v)_{x}=0$. По предложению 4 коэффициенты $U_{i j}=r v_{i j}$ однозначно определяются начальными данньми $r_{0}(x) v_{i j}(0, x)=\left(U_{0}\right)_{i j}(x)$. Таким образом, сильное о.э.p. $U(t, x)$ задачи $(4),(42)$ единственно.

ЗАмЕчАНИЕ 4 . Пусть $u=u(t, x)$ - сильное о.э.р. задачи (1), (2). Как установлено при доказательстве теоремы 6, для любой однородной функции $h(u) \in C\left(\mathbb{R}^{n}\right)$ вьполнено равенство (39). В частности, если рассмотреть замену переменных $v=h(u) \in \Omega \subset \mathbb{R}^{m}$ с однородньми компонентами такую, что $r=|u|$ является функцией новых переменных: $r=r(v)$, то вектор $v=h(u(t, x))$ удовлетворяет системе

$$
v_{t}+(\varphi(r) v)_{x}=0, \quad r=r(v), \quad v \in \Omega
$$

в $\mathscr{D}^{\prime}\left(\Pi, \mathbb{R}^{m}\right)$ и начальным данным

$$
v(0, x)=v_{0}(x)=h\left(u_{0}(x)\right)
$$

Для задачи Коши (43), (44) можно ввести определение сильного о.э.р., аналогичное определению 5 , и так же, как и для задачи (1), (2), установить его сушествование и единственность. Рассуждения, приведенные выше, показывают, что для сильного о.э.p. $u=u(t, x)$ задачи $(1),(2)$ вектор-функция $v=h(u)$ будет сильньм о.э.р. задачи $(43),(44)$.

Если $\|\cdot\|$ - некоторая норма на $\mathbb{R}^{n}$, то однородная невырожденная замена $v=$ $h(u)=|u| \cdot u /\|u\|$ (при $u=0$ считаем, что $v=0$ ) приводит к задаче (43), (44) с $r=\|v\|$. Таким образом, теория сильных о.э.р. задачи (1), (2) обобщается на 
случай, когда вместо $|\cdot|$ в (1) берется произвольная норма. Нетрудно показать, что основные результаты теории остаются справедливыми и для бесконечномерных систем такого вида, например, когда $u$ принимает значения в рефлексивном банаховом пространстве.

Заметим также, что если $v=h(u)=\left(u_{1}^{2} / r, \ldots, u_{n}^{2} / r\right)$ (при $r=0$ полагаем $h(u)=0)$, то $r=v_{1}+\cdots+v_{n}$ и для $\varphi(r)=1 /(1+r)$ система (43) является известной системой многокомпонентной хроматографии:

$$
v_{t}+\left(\frac{v}{1+r}\right)_{x}=0, \quad r=v_{1}+\cdots+v_{n}, \quad v_{i} \geqslant 0, \quad i=1, \ldots, n
$$

ЗАмЕчАнИЕ 5. Известно (см. принцип сравнения из [8]-[10]), что о.э.p. $r(t, x)$ скалярной задачи $(23),(24)$ является наибольшим о.э.суб-р. этой задачи. Из определения 5 и теоремы 3 легко выводится тогда следующий экстремальный принцип:

сильное о.э.p. $u(t, x)$ задачи $(1),(2)$ является о.э.р. этой задачи, для которого значение функционала $\int|u(t, x)| e^{-t-|x|} d t d x$ максимально.

\section{§4. Обоснование метода "исчезающей вязкости"}

На протяжении параграфа будем предполагать, что функция $f(r)=\varphi(|r|) r \in$ $C^{1}(\mathbb{R})$ и нелинейна на невырожденных интервалах. Выберем последовательность $\varepsilon_{k}>0, \varepsilon_{k} \rightarrow 0$ при $k \rightarrow \infty$, и рассмотрим последовательность $u_{k}=u_{k}(t, x) \in$ $C^{2}\left(\Pi, \mathbb{R}^{n}\right) \cap L^{\infty}\left(\Pi, \mathbb{R}^{n}\right)$ решений задачи Коши для параболической системы (18) при $\varepsilon=\varepsilon_{k}$ с начальными данными $u_{0 k}(x)$. Допустим также, что последовательность $u_{0 k}$ ограничена в $L^{\infty}\left(\mathbb{R}, \mathbb{R}^{n}\right), u_{0 k} \rightarrow u_{0}$ в $L_{\text {lос }}^{1}\left(\mathbb{R}, \mathbb{R}^{n}\right)$ и для вектор-функции $u_{0}(x)$ вьполнено условие $(\mathrm{C})$. Целью настояшего параграфа является доказательство того, что при сделанных предположениях последовательность $u_{k}$ сходится в $L_{\text {loc }}^{1}\left(\Pi, \mathbb{R}^{n}\right)$ к сильному о.э.р. исходной задачи $(1),(2)$. Мы будем также считать, что в условии $(\mathrm{C})$ вектор $e=(1,0, \ldots, 0)$ - первый базисный вектор, чего всегда можно добиться с помощью ортогональной замены $u \mapsto T u$ (см. замечание 2 ).

Установим прежде всего следующий принцип максимума.

ПРЕДЛОЖЕНИЕ 7. Пусть $\varepsilon>0, u=u(t, x) \in C^{2}\left(\Pi, \mathbb{R}^{n}\right) \cap L^{\infty}\left(\Pi, \mathbb{R}^{n}\right)-$ решение задачи Коши (18), (2). Тогда $|u(t, x)| \leqslant M=\operatorname{ess} \sup _{x \in \mathbb{R}}\left|u_{0}(x)\right| \partial \Omega_{\text {я }}$ $(t, x) \in \Pi$.

ДокаЗАТЕЛЬСтво. Обозначим $M_{1}=|u|_{\infty}=\operatorname{ess}_{\sup }(t, x) \in \Pi|u(t, x)|$. Пусть $\eta_{m}(r)=r^{m}, \psi_{m}(r)=\int_{0}^{r} f^{\prime}(s) \eta_{m}^{\prime}(s) d s$ при $m>2$. Если $N=\max _{\left[0, M_{1}\right]}\left|f^{\prime}(r)\right|$, то при $r \in\left[0, M_{1}\right]$ с учетом равенства $\eta_{m}^{\prime}(s)=m s^{m-1}$ имеем оценку

$$
\left|\psi_{m}(r)\right| \leqslant N \int_{0}^{r} \eta_{m}^{\prime}(s) d s=N \eta_{m}(r)
$$

По теореме 1 и предложению 1 функция $p_{m}(u)=\eta_{m}(|u|)$ является выпуклой энтропией системы (1) с потоком $q_{m}(u)=\psi_{m}(|u|)$. В силу леммы 2 тогда

$$
p_{m}(u)_{t}+q_{m}(u)_{x} \leqslant \varepsilon p_{m}(u)_{x x} \quad \text { в } \quad \mathscr{D}^{\prime}(\Pi) .
$$


Выберем функцию $h(x) \in C^{2}(\mathbb{R})$ так, что $\left|h^{\prime}(x)\right| \leqslant 1,\left|h^{\prime \prime}(x)\right| \leqslant 1$ всюду на $\mathbb{R}$, $h(x)=|x|$ при $|x| \geqslant 1$, и положим $g(x)=e^{-h(x)}$. Тогда $g(x) \in C^{2}(\mathbb{R}) \cap L^{1}(\mathbb{R})$, $g(x)>0$ и

$$
\begin{aligned}
\left|g^{\prime}(x)\right| & =\left|h^{\prime}(x)\right| g(x) \leqslant g(x), \\
\left|g^{\prime \prime}(x)\right| & \leqslant\left(\left(h^{\prime}(x)\right)^{2}+\left|h^{\prime \prime}(x)\right|\right) g(x) \leqslant 2 g(x) .
\end{aligned}
$$

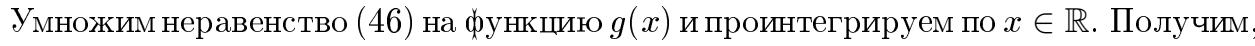
что в $\mathscr{D}^{\prime}\left(\mathbb{R}_{+}\right)$

$$
\frac{d}{d t} \int p_{m}(u(t, x)) g(x) d x \leqslant \int q_{m}(u(t, x)) g^{\prime}(x) d x+\varepsilon \int p_{m}(u(t, x)) g^{\prime \prime}(x) d x
$$

откуда с учетом оценок (47)

$$
\frac{d}{d t} \int p_{m}(u(t, x)) g(x) d x \leqslant \int\left|q_{m}(u(t, x))\right| g(x) d x+2 \varepsilon \int p_{m}(u(t, x)) g(x) d x
$$

Поскольку $|u(t, x)| \leqslant M_{1}$, то $\left|q_{m}(u(t, x))\right| \leqslant N p_{m}(u(t, x))$ ввиду (45), и из (48) вытекает, что функция $F_{m}(t)=\int p_{m}(u(t, x)) g(x) d x$ удовлетворяет соотношению $F_{m}^{\prime}(t) \leqslant C \cdot F_{m}(t), C=(N+2 \varepsilon)$.

По лемме Гронуолла $F_{m}(t) \leqslant F_{m}(0) e^{C t}$, где

$$
F_{m}(0)=\int p_{m}\left(u_{0}(x)\right) g(x) d x=\int\left|u_{0}(x)\right|^{m} g(x) d x \leqslant \text { const } \cdot M^{m}
$$

ввиду условия $\left|u_{0}(x)\right| \leqslant M$ п.в. на $\mathbb{R}$. Итак, $F_{m}(t) \leqslant \mathrm{const} \cdot e^{C t} M^{m} \forall t>0$, откуда

$$
\limsup _{m \rightarrow \infty}\left(F_{m}(t)\right)^{1 / m} \leqslant M
$$

Как легко видеть, существует предел

$$
\lim _{m \rightarrow \infty}\left(F_{m}(t)\right)^{1 / m}=\underset{x \in \mathbb{R}}{\operatorname{ess} \sup }|u(t, x)|
$$

Из (49) вытекает тогда требуемая оценка $|u(t, x)| \leqslant M$. Предложение доказано.

Заметим, что при достаточно гладких функции $f(u)$ и начальных данных действительно сушествует единственное классическое решение задачи (18), (2). С учетом предложения 7 это следует из результатов монографии [13].

Нам потребуется далее понятие мерозначной функции. Пусть $\Omega$ - некоторая область в $\mathbb{R}^{N}$. Мерозначная функиия со значениями в $\mathbb{R}^{n}$ - это слабо измеримое отображение $x \rightarrow \nu_{x}$ области $\Omega$ в пространство $\operatorname{Prob}_{0}\left(\mathbb{R}^{n}\right)$ вероятностных борелевских мер с компактным носителем на $\mathbb{R}^{n}$. Слабая измеримость $\nu_{x}$ означает, что $\forall p(\xi) \in C\left(\mathbb{R}^{n}\right)$ функция $x \mapsto \int_{\mathbb{R}^{n}} p(\xi) d \nu_{x}(\xi)$ измерима на $\Omega$.

Мерозначная функция $\nu_{x}$ называется ограниченной, если существует $M>0$ такое, что для почти всех $x \in \Omega \operatorname{supp} \nu_{x}$ лежит в шаре $|\xi| \leqslant M$. Минимальное из таких $M$ будем обозначать $\left\|\nu_{x}\right\|_{\infty}$. 
Наконец, мерозначные функции вида $\nu_{x}(\xi)=\delta(\xi-u(x)), u(x) \in L^{\infty}\left(\Omega, \mathbb{R}^{n}\right)$ (где $\delta(\xi-u)$ - мера Дирака в точке $\left.u \in \mathbb{R}^{n}\right)$, называются регулярными и отождествляются с соответствующими функциями $u(x)$. Таким образом, имеется естественное вложение $L^{\infty}\left(\Omega, \mathbb{R}^{n}\right) \subset \operatorname{MV}\left(\Omega, \mathbb{R}^{n}\right)$, где через $\operatorname{MV}\left(\Omega, \mathbb{R}^{n}\right)$ обозначено множество ограниченных мерозначных функций на $\Omega$ со значениями в $\mathbb{R}^{n}$.

Мерозначные функции естественно возникают как слабые пределы ограниченных последовательностей из $L^{\infty}\left(\Omega, \mathbb{R}^{n}\right)$ в смысле следуюшего результата Тартара (см. [14]).

ПРЕДЛОЖЕНИЕ 8. Пусть $u_{k}(x), k \in \mathbb{N},-$ ограниченная в $L^{\infty}\left(\Omega, \mathbb{R}^{n}\right)$ nоследовательность, $\left\|u_{k}\right\|_{\infty} \leqslant M$. Тогда существует ограниченная мерозначная функиия $\nu_{x} \in \operatorname{MV}\left(\Omega, \mathbb{R}^{n}\right),\left\|\nu_{x}\right\|_{\infty} \leqslant M$, и подпоследовательность $u_{r}(x)=u_{k_{r}}(x)$ последовательности $u_{k}$ такие, что $\forall p(\xi) \in C\left(\mathbb{R}^{n}\right)$

$$
p\left(u_{r}\right) \underset{r \rightarrow \infty}{\longrightarrow} \int_{\mathbb{R}^{n}} p(\xi) d \nu_{x}(\xi) \quad \text { *-слабо } \quad \text { в } L^{\infty}(\Omega)
$$

при этом мерозначная функиия $\nu_{x}$ регулярна: $\nu_{x}(\xi)=\delta(\xi-u), u=u(x) \in$ $L^{\infty}\left(\Omega, \mathbb{R}^{n}\right)$, тогда и только тогда, когда $u_{r} \rightarrow$ и в $L_{\mathrm{loc}}^{1}\left(\Omega, \mathbb{R}^{n}\right)($ сильно $)$.

В дополнение к предложению 8 заметим, что, обратно, любая ограниченная мерозначная функция может быть получена как предел ограниченной последовательности из $L^{\infty}\left(\Omega, \mathbb{R}^{n}\right)$ в смысле предельного соотношения (50).

Предложение 8 позволяет осушествить предельньй переход по последовательности решений $u_{k}$ параболической задачи $(18),(2)$, используя лишь содержашуюся в предложении 7 априорную оценку $\left|u_{k}\right| \leqslant M$. В результате такого предельного перехода получим ограниченную мерозначную функцию $\nu_{t, x} \in \mathrm{MV}\left(\Pi, \mathbb{R}^{n}\right)$ такую, что $\left\|\nu_{t, x}\right\|_{\infty} \leqslant M$ и (возможно, после выделения подпоследовательности, за которой мы сохраним прежнее обозначение $\left.u_{k}\right) \forall p(u) \in C\left(\mathbb{R}^{n}\right)$

$$
p\left(u_{k}\right) \underset{k \rightarrow \infty}{\longrightarrow} \int_{\mathbb{R}^{n}} p(u) d \nu_{t, x}(u) \text { *-слабо в } L^{\infty}(\Pi) .
$$

Для предельной мерозначной функции $\nu_{t, x}$ справедлива следуюшая

ТЕОРема 8. Пусть $p(u)$ - выпуклая энтропия системы (1), $q(u)$ - соответствующий поток. Тогда $\forall g=g(t, x) \in C_{0}^{\infty}(\bar{\Pi}), g(t, x) \geqslant 0$,

$$
\begin{aligned}
\int_{\Pi}\left[\left(\int_{\mathbb{R}^{n}} p(u) d \nu_{t, x}(u)\right) g_{t}+\left(\int_{\mathbb{R}^{n}} q(u) d \nu_{t, x}(u)\right) g_{x}\right] d t d x & \\
& +\int_{\mathbb{R}} p\left(u_{0}(x)\right) g(0, x) d x \geqslant 0
\end{aligned}
$$

ДокАЗАТЕЛьство. По лемме 2 для всех $k \in \mathbb{N}$

$$
p\left(u_{k}\right)_{t}+q\left(u_{k}\right)_{x} \leqslant \varepsilon_{k} p\left(u_{k}\right)_{x x} \quad \text { в } \mathscr{D}^{\prime}(\Pi) .
$$


Умножив это неравенство на неотрицательную пробную функцию $g=g(t, x) \in$ $C_{0}^{\infty}(\bar{\Pi})$ и проинтегрировав по частям, получим, что

$$
\int_{\Pi}\left[p\left(u_{k}\right) g_{t}+q\left(u_{k}\right) g_{x}+\varepsilon_{k} p\left(u_{k}\right) g_{x x}\right] d t d x+\int_{\mathbb{R}} p\left(u_{0 k}(x)\right) g(0, x) d x \geqslant 0
$$

откуда в пределе при $k \rightarrow \infty$ с учетом (51), предельного соотношения $u_{0 k}(x) \rightarrow u_{0}$ в $L_{\text {loc }}^{1}\left(\mathbb{R}, \mathbb{R}^{n}\right)$ и условия $\varepsilon_{k} \rightarrow 0$ получим соотношение (52). Теорема доказана.

Выбирая в (52) неотрицательные пробные функции из $C_{0}^{\infty}(\Pi)$, получаем, что для выпуклых энтропий $p(u)$

$$
\left(\int_{\mathbb{R}^{n}} p(u) d \nu_{t, x}(u)\right)_{t}+\left(\int_{\mathbb{R}^{n}} q(u) d \nu_{t, x}(u)\right)_{x} \leqslant 0 \quad \text { в } \mathscr{D}^{\prime}(\Pi),
$$

т.е. мерозначная функция $\nu_{t, x}$ удовлетворяет энтропийному условию (16) “в среднем". Из соотношения (53) с линейнњми энтропиями $p(u)= \pm(c, u)$ вытекает, в частности, равенство

$$
\left(\int_{\mathbb{R}^{n}} u d \nu_{t, x}(u)\right)_{t}+\left(\int_{\mathbb{R}^{n}} \varphi(|u|) u d \nu_{t, x}(u)\right)_{x}=0 \quad \text { в } \quad \mathscr{D}^{\prime}\left(\Pi, \mathbb{R}^{n}\right)
$$

которое означает, что $\nu_{t, x}$ удовлетворяет "в среднем" системе $(1)$.

Из (52) можно получить также начальное условие $\nu_{0, x}(u)=\delta\left(u-u_{0}(x)\right)$ в смысле следующего предложения.

ПРЕДЛОЖЕНИЕ 9. Справедливо предельное соотношение

$$
\underset{t \rightarrow 0+}{\operatorname{ess} \lim _{t \rightarrow}} \int\left|u-u_{0}(x)\right| d \nu_{t, x}(u)=0 \quad \text { в } \quad L_{\mathrm{loc}}^{1}(\mathbb{R})
$$

ДоказАтЕльство. Обозначим через $\mathscr{A}$ множество, состоящее из значений $t>$ 0, являющихся точками Лебега функций

$$
t \mapsto F(t, p, h)=\int_{\mathbb{R}}\left(\int_{\mathbb{R}^{n}} p(u) d \nu_{t, x}(u)\right) h(x) d x
$$

для всех $p(u) \in C\left(\mathbb{R}^{n}\right), h(x) \in L^{1}(\mathbb{R})$. Из сепарабельности пространств $C\left(\mathbb{R}^{n}\right)$, $L^{1}(\mathbb{R})$ и непрерьвной зависимости $F(t, p, h)$ от $p(u) \in C\left(\mathbb{R}^{n}\right), h(x) \in L^{1}(\mathbb{R})$ легко следует, что $\mathscr{A}$ - множество полной меры в $\mathbb{R}_{+}$. Выберем функцию $\gamma(s) \in C_{0}^{\infty}(\mathbb{R})$, $\gamma(s) \geqslant 0, \operatorname{supp} \gamma \subset[0,1], \int \gamma(s) d s=1$, и положим $\delta_{k}(s)=k \gamma(k s)$ при $k \in \mathbb{N}$. Ясно, что $\delta_{k} \in C_{0}^{\infty}(\mathbb{R}), \delta_{k} \geqslant 0, \operatorname{supp} \delta_{k} \subset[0,1 / k], \int \delta_{k}(s) d s=1$ (так что при $k \rightarrow \infty$ последовательность $\delta_{k}$ сходится в $\mathscr{D}^{\prime}(\mathbb{R})$ к $\delta$-функции Дирака). Определим далее последовательность $\chi_{k}(t)=\int_{-\infty}^{t} \delta_{k}(s) d s$, сходящуюся при $k \rightarrow \infty \mathrm{k}$ характеристической функции интервала $(0,+\infty)$. 
Пусть $\tau \in \mathscr{A}, h(x) \in C_{0}^{\infty}(\mathbb{R}), h(x) \geqslant 0$. Возьмем в соотношении $(52) g(t, x)=$ $h(x)\left(1-\chi_{k}(t-\tau)\right)$. Получим после элементарных преобразований, что

$$
\begin{aligned}
& -\int_{\Pi}\left(\int_{\mathbb{R}^{n}} p(u) d \nu_{t, x}(u)\right) h(x) \delta_{k}(t-\tau) d t d x \\
& +\int_{\Pi}\left(\int_{\mathbb{R}^{n}} q(u) d \nu_{t, x}(u)\right) h^{\prime}(x) \chi_{k}(t-\tau) d t d x \\
& \quad+\int_{\mathbb{R}} p\left(u_{0}(x)\right) h(x) d x \geqslant 0 .
\end{aligned}
$$

Из (56) с учетом того, что $\tau$ - точка Лебега функции

$$
\int_{\mathbb{R}}\left(\int_{\mathbb{R}^{n}} p(u) d \nu_{t, x}(u)\right) h(x) d x
$$

в пределе при $k \rightarrow \infty$ следует соотношение

$$
\begin{aligned}
& \int_{\mathbb{R}}\left(\int_{\mathbb{R}^{n}} p(u) d \nu_{\tau, x}(u)-p\left(u_{0}(x)\right)\right) h(x) d x \\
& \quad \leqslant \int_{[0, \tau] \times \mathbb{R}}\left(\int_{\mathbb{R}^{n}}|q(u)| d \nu_{t, x}(u)\right)\left|h^{\prime}(x)\right| d t d x \leqslant \mathrm{const} \cdot\left\|h^{\prime}\right\|_{1} \tau
\end{aligned}
$$

откуда

$$
\limsup _{\substack{\tau \rightarrow 0+\\ \tau \in \mathscr{A}}} \int_{\mathbb{R}}\left(\int_{\mathbb{R}^{n}} p(u) d \nu_{\tau, x}(u)-p\left(u_{0}(x)\right)\right) h(x) d x \leqslant 0 .
$$

Взяв в $(57)$ линейные энтропии $p(u)= \pm(c, u)$, получим, что $\forall h(x) \in C_{0}^{\infty}(\mathbb{R})$, $h(x) \geqslant 0$,

$$
\lim _{\substack{\tau \rightarrow 0+\\ \tau \in \mathscr{A}}} \int_{\mathbb{R}}\left(\int_{\mathbb{R}^{n}} u d \nu_{\tau, x}(u)-u_{0}(x)\right) h(x) d x=0 .
$$

Поскольку линейная оболочка неотрицательных функций из $C_{0}^{\infty}(\mathbb{R})$ плотна в $L^{1}(\mathbb{R})$, то из $(58)$ следует, что

$$
\int_{\mathbb{R}^{n}} u d \nu_{\tau, x}(u) \underset{\substack{\tau \rightarrow 0+\\ \tau \in \mathscr{A}}}{\longrightarrow} u_{0}(x) \quad \text { *-слабо в } L^{\infty}\left(\mathbb{R}, \mathbb{R}^{n}\right)
$$

Далее из (57) при $p(u)=|u|^{2}$ вытекает соотношение

$$
\limsup _{\substack{\tau \rightarrow 0+\\ \tau \in \mathscr{A}}} \int_{\mathbb{R}}\left(\int_{\mathbb{R}^{n}}|u|^{2} d \nu_{\tau, x}(u)\right) h(x) d x \leqslant \int_{\mathbb{R}}\left|u_{0}(x)\right|^{2} h(x) d x
$$

и ввиду (59) (здесь, как обычно, $(\cdot, \cdot)$ - скалярное произведение в $\mathbb{R}^{n}$ )

$$
\lim _{\substack{\tau \rightarrow 0+\\ \tau \in \mathscr{A}}} \int_{\mathbb{R}}\left(\int_{\mathbb{R}^{n}}\left(u, u_{0}(x)\right) d \nu_{\tau, x}(u)\right) h(x) d x=\int_{\mathbb{R}}\left|u_{0}(x)\right|^{2} h(x) d x .
$$


С учетом этих соотношений

$$
\begin{aligned}
& \underset{\substack{\tau \rightarrow 0+\\
\tau \in \mathscr{A}}}{\limsup _{\mathbb{R}}} \int_{\mathbb{R}}\left(\int_{\mathbb{R}^{n}}\left|u-u_{0}(x)\right|^{2} d \nu_{\tau, x}(u)\right) h(x) d x \\
& =\int_{\mathbb{R}}\left|u_{0}(x)\right|^{2} h(x) d x+\underset{\substack{\tau \rightarrow 0+\\
\tau \in \mathscr{A}}}{\limsup }\left(\int_{\mathbb{R}}\left(\int_{\mathbb{R}^{n}}|u|^{2} d \nu_{\tau, x}(u)\right) h(x) d x\right. \\
& \left.\quad-2 \int_{\mathbb{R}^{\prime}}\left(\int_{\mathbb{R}^{n}}\left(u, u_{0}(x)\right) d \nu_{\tau, x}(u)\right) h(x) d x\right)=0,
\end{aligned}
$$

откуда ввиду произвольности $h(x) \in C_{0}^{\infty}(\mathbb{R}), h(x) \geqslant 0$, и следует (55). Предложение доказано.

Соотношения $(53),(55)$ означают, что $\nu_{t, x}$ является мерозначным решением (коротко-м.р.) задачи Коши (1), (2) в смысле ДиПерна [15]. Ниже мы установим, что м.р. $\nu_{t, x}$ регулярно: $\nu_{t, x}(u)=\delta(u-u(t, x)), u(t, x) \in L^{\infty}\left(\Pi, \mathbb{R}^{n}\right)$. В силу предложения 8 отсюда будет следовать сильная сходимость последовательности $u_{k}$ к $u=u(t, x)$.

Пусть $H^{-1}$ - пространство Соболева $H_{2}^{-1}\left(\mathbb{R}^{2}\right)$ (определение и основные свойства пространств Соболева $H_{p}^{s}$ с произвольными вещественными показателями $s$ можно найти, например, в монографии [16]). Обозначим через $H_{\mathrm{loc}}^{-1}(\Pi)$ пространство, состоящее из распределений $u \in \mathscr{D}^{\prime}(\Pi)$ таких, что $u g \in H^{-1}$ для всех $g \in C_{0}^{\infty}(\Pi)$. Топология на $H_{\mathrm{loc}}^{-1}(\Pi)$ задается полунормами $\|u g\|_{H^{-1}}, g \in C_{0}^{\infty}(\Pi)$.

ПРЕДЛОЖЕНИЕ 10. ДЛя любой выпуклой энтропии $p(u)$ и соответствующего потока $q(u)$ последовательность распределений $p\left(u_{k}\right)_{t}+q\left(u_{k}\right)_{x}$ предкомпактна в $H_{\mathrm{loc}}^{-1}(\Pi)$.

ДокАЗАтЕльство. Заметим, что последовательность распределений $l_{k}=$ $p\left(u_{k}\right)_{t}+q\left(u_{k}\right)_{x}$ ограничена в $H_{\infty}^{-1}(\Pi)$ ввиду ограниченности в $L^{\infty}(\Pi)$ функций $p\left(u_{k}\right), q\left(u_{k}\right)$. Из результатов Мюра (см. [14; лемма 28]) тогда следует, что для доказательства предложения достаточно найти представление $l_{k}=\alpha_{k}-\beta_{k}$, в котором последовательность $\alpha_{k}$ предкомпактна в $H_{\mathrm{loc}}^{-1}(\Pi)$, а $\beta_{k}$ ограничена в $\mathscr{M}(\Pi)$, где $\mathscr{M}(\Pi)$ - пространство локально конечных борелевских мер на П с топологией, порожденной полунормами $\beta \mapsto \operatorname{var}(g \beta), g \in C_{0}^{\infty}(\Pi)$.

Положим $l_{k}=\alpha_{k}-\beta_{k}$, где $\alpha_{k}=\varepsilon_{k} p\left(u_{k}\right)_{x x}$, и покажем, что данное представление удовлетворяет требуемым условиям. По лемме 2 распределение $\beta_{k} \geqslant 0$ в $\mathscr{D}^{\prime}(\Pi)$ и, значит, является неотрицательной локально конечной борелевской мерой на П. Если $g=g(t, x) \in C_{0}^{\infty}(\Pi), g \geqslant 0$, то из равенства $-\beta_{k}=p\left(u_{k}\right)_{t}+q\left(u_{k}\right)_{x}-$ $\varepsilon_{k} p\left(u_{k}\right)_{x x}$ следует, что

$$
\left\langle\beta_{k}, g\right\rangle=\int_{\Pi} g(t, x) d \beta_{k}(t, x)=\int_{\Pi}\left[p\left(u_{k}\right) g_{t}+q\left(u_{k}\right) g_{x}+\varepsilon_{k} p\left(u_{k}\right) g_{x x}\right] d t d x
$$

и с учетом оценки $\left|u_{k}\right| \leqslant M$ величина $\operatorname{var}\left(g \beta_{k}\right)=\int_{\Pi} g(t, x) d \beta_{k}(t, x)$ ограничена по $k \in \mathbb{N}$. Это означает ограниченность последовательности $\beta_{k}$ в пространстве $\mathscr{M}(\Pi)$. Покажем далее, что при $k \rightarrow \infty$ последовательность $\alpha_{k} \rightarrow 0$ в $H_{\mathrm{loc}}^{-1}(\Pi)$. По лемме $1 p\left(u_{k}\right)_{x}=\left(\nabla p\left(u_{k}\right),\left(u_{k}\right)_{x}\right)$ и

$$
\alpha_{k}=\varepsilon_{k} p\left(u_{k}\right)_{x x}=\sqrt{\varepsilon_{k}}\left(\nabla p\left(u_{k}\right), \sqrt{\varepsilon_{k}}\left(u_{k}\right)_{x}\right)_{x} .
$$


Поэтому для доказательства того, что $\alpha_{k} \rightarrow 0$ в $H_{\mathrm{loc}}^{-1}(\Pi)$, достаточно установить ограниченность последовательности $\sqrt{\varepsilon_{k}}\left(u_{k}\right)_{x}$ в $L_{\mathrm{loc}}^{2}\left(\Pi, \mathbb{R}^{n}\right)$. Для этого возьмем энтропию $p_{0}(u)=|u|^{2} / 2$, пусть $q_{0}(u)$ - соответствующий поток. Умножая равенство $\left(u_{k}\right)_{t}+A\left(u_{k}\right)\left(u_{k}\right)_{x}=\varepsilon_{k}\left(u_{k}\right)_{x x}$ скалярно на $\nabla p_{0}\left(u_{k}\right)=u_{k}$, получаем соотношение

$$
p_{0}\left(u_{k}\right)_{t}+q_{0}\left(u_{k}\right)_{x}=\varepsilon_{k} p_{0}\left(u_{k}\right)_{x x}-\varepsilon_{k}\left|\left(u_{k}\right)_{x}\right|^{2},
$$

из которого путем умножения на неотрицательную пробную функцию $g=g(t, x) \in$ $C_{0}^{\infty}(\Pi)$ и интегрирования по частям получается равенство

$$
\varepsilon_{k} \int_{\Pi}\left|\left(u_{k}\right)_{x}\right|^{2} g(t, x) d t d x=\int_{\Pi}\left[p_{0}\left(u_{k}\right) g_{t}+q_{0}\left(u_{k}\right) g_{x}+\varepsilon_{k} p_{0}\left(u_{k}\right) g_{x x}\right] d t d x
$$

Учитывая, что $\left|u_{k}\right| \leqslant M$, приходим к оценке

$$
\varepsilon_{k} \int_{\Pi}\left|\left(u_{k}\right)_{x}\right|^{2} g(t, x) d t d x \leqslant c
$$

где $c=c(g)$ - константа, не зависящая от $k$. Это как раз и означает, что последовательность $\sqrt{\varepsilon_{k}}\left(u_{k}\right)_{x}$ ограничена в $L_{\text {loc }}^{2}\left(\Pi, \mathbb{R}^{n}\right)$. Мы установили, что последовательность $\alpha_{k} \rightarrow 0$ и, значит, предкомпактна в $H_{\text {loc }}^{-1}(\Pi)$. Предложение доказано.

Из предложения 10 в соответствии с теорией компенсированной компактности вытекают следующие коммутационные соотношения Тартара-Мюра (см. [14], [17]): для почти всех $(t, x) \in \Pi$

$$
\begin{aligned}
\int_{\mathbb{R}^{n}} & {\left[p_{1}(u) q_{2}(u)-p_{2}(u) q_{1}(u)\right] d \nu_{t, x}(u) } \\
= & \int_{\mathbb{R}^{n}} p_{1}(u) d \nu_{t, x}(u) \int_{\mathbb{R}^{n}} q_{2}(u) d \nu_{t, x}(u) \\
& -\int_{\mathbb{R}^{n}} p_{2}(u) d \nu_{t, x}(u) \int_{\mathbb{R}^{n}} q_{1}(u) d \nu_{t, x}(u),
\end{aligned}
$$

справедливые для любой пары выпуклых энтропий $p_{1}, p_{2}$ с соответствующими потоками $q_{1}, q_{2}$. Эти соотношения позволяют установить следующий результат.

Теорема 9. Для почти всех $(t, x) \in \Pi$ функция $r=|u|$ постоянна на замкнутом носителе $\operatorname{supp} \nu_{t, x}$ мерь $\nu_{t, x}(u):|u|=r(t, x)$.

ДокАЗАТЕЛЬство. Пусть $\tilde{\nu}_{t, x} \in \mathrm{MV}(\Pi, \mathbb{R})$ - скалярная мерозначная функция, определенная условием $\tilde{\nu}_{t, x}=r^{*} \nu_{t, x}$, где $r^{*} \nu_{t, x}$ обозначает образ меры $\nu_{t, x}$ при отображении $u \mapsto r=|u|$. Ясно, что для доказательства теоремы нужно установить регулярность $\tilde{\nu}_{t, x}$. Из (60) при $p_{1}(u)=\eta_{1}(r), p_{2}(u)=\eta_{2}(r)$, где $r=|u|$, $\eta_{1}(r), \eta_{2}(r) \in C^{1}(\mathbb{R})$ - неубывающие вьпуклые функции, следует коммутационное соотношение: для почти всех $(t, x) \in \Pi$

$$
\begin{aligned}
\int_{\mathbb{R}}[ & \left.\eta_{1}(r) \psi_{2}(r)-\eta_{2}(r) \psi_{1}(r)\right] d \tilde{\nu}_{t, x}(r) \\
= & \int_{\mathbb{R}} \eta_{1}(r) d \tilde{\nu}_{t, x}(r) \int_{\mathbb{R}} \psi_{2}(r) d \tilde{\nu}_{t, x}(r) \\
& -\int_{\mathbb{R}} \eta_{2}(r) d \tilde{\nu}_{t, x}(r) \int_{\mathbb{R}} \psi_{1}(r) d \tilde{\nu}_{t, x}(r),
\end{aligned}
$$


$\psi_{i}^{\prime}(r)=f^{\prime}(r) \eta_{i}^{\prime}(r), i=1,2$. Так как линейная оболочка неубьвающих вьпуклых функций плотна в $C^{1}(\mathbb{R})$, то $(61)$ выполнено для всех $\eta_{1}(r), \eta_{2}(r) \in C^{1}(\mathbb{R})$. Как показано в [14], при нашем предположении о нелинейности функции $f$ на невырожденных интервалах из условия (61) вытекает регулярность $\tilde{\nu}_{t, x}$ (последнее верно и для лишш непрерывной функции $f$; общий многомерный аналог этого утверждения доказан, например, в [18]). Таким образом, для почти всех $(t, x) \in \Pi$ $\tilde{\nu}_{t, x}(r)=\delta(r-r(t, x)), r(t, x) \in L^{\infty}(\Pi)$. Теорема доказана.

Из теоремы 9 следует, что п.в. на П носители мер $\nu_{t, x}$ лежат на сферах $|u|=$ $r(t, x)$. Заметим также, что, обратно, для таких мерозначных функций $\nu_{t, x}$ выполнены условия (60) для всех пар допустимых энтропий вида (12). Поэтому из метода компенсированной компактности нельзя извлечь большей информации, чем содержится в теореме 9. Для доказательства регулярности $\nu_{t, x}$ необходимо использовать также регулярность начальных данных и условие $(\mathrm{C})$. Из условия $(\mathrm{C})$ вытекает следуюшее ограничение на носители мер $\nu_{t, x}$, являюшееся мерозначным аналогом теоремы 4 (при $\delta=0)$.

ПРЕДЛОЖЕНИЕ 11. ДЛя почти всеx $(t, x) \in \Pi \operatorname{supp} \nu_{t, x} \subset \dot{\Gamma}(e, 0)$, m.e. $u_{1}>0 н a \operatorname{supp} \nu_{t, x}$ npu $r(t, x)>0$.

Доказательство аналогично доказательству теоремы 4 при $\delta=0$. Просто нужно энтропийное условие (16) заменить на мерозначньй аналог этого условия (53), так что всюду в доказательстве величины вида $p(u(t, x))$ заменятся интегральными средними $\int_{\mathbb{R}^{n}} p(u) d \nu_{t, x}(u)$.

Мы готовы приступить к доказательству основной теоремы.

Tеорема 10. Мерозначное решение $\nu_{t, x}$ регулярно: $\nu_{t, x}(u)=\delta(u-u(t, x))$, где $и(t, x)$ - сильное о.э.р. задачи (1), (2). При этом последовательность $u_{k}(t, x)$ сходится при $k \rightarrow \infty \kappa u(t, x)$ в $L_{\mathrm{loc}}^{1}\left(\Pi, \mathbb{R}^{n}\right)$.

ДокАЗАТЕЛЬСтво. Рассмотрим при $r=r(u)=|u|$ вектор

$$
v= \begin{cases}\frac{u}{r}, & r>0 \\ 0, & r=0\end{cases}
$$

По теореме $9 r(u)=r(t, x)$ на носителе меры $\nu_{t, x}(u)$ для почти всех $(t, x)$, и мы можем переписать равенство (54) в виде:

$$
\left(r \int_{\mathbb{R}^{n}} v d \nu_{t, x}(u)\right)_{t}+\left(f(r) \int_{\mathbb{R}^{n}} v d \nu_{t, x}(u)\right)_{x}=0 \quad \text { в } \quad \mathscr{D}^{\prime}\left(\Pi, \mathbb{R}^{n}\right) .
$$

Обозначим $I_{k}=I_{k}(t, x)=\int_{\mathbb{R}^{n}} v_{k} d \nu_{t, x}(u), k=1, \ldots, n$. Из предложения 11 непосредственно следует, что $I_{1}>0$ при $r=r(t, x) \neq 0$. Пусть

$$
\begin{aligned}
& A=A(t, x)=r I_{1}=\int_{\mathbb{R}^{n}} u_{1} d \nu_{t, x}(u) \\
& B=B(t, x)=f(r) I_{1}=\int_{\mathbb{R}^{n}} \varphi(r) u_{1} d \nu_{t, x}(u)
\end{aligned}
$$


Так же как при доказательстве теоремы 7 устанавливается, что для функций $A, B$ выполнены условия (32)-(34). Здесь нужно учесть (62) и предельное соотношение

$$
\underset{t \rightarrow 0+}{\operatorname{ess}} \lim A(t, x)=A(0, x)=u_{01}(x) \quad \text { в } \quad L_{\mathrm{loc}}^{1}(\mathbb{R}),
$$

следующее из начального условия (55). По (62) $\left(A \theta_{k}\right)_{t}+\left(B \theta_{k}\right)_{x}=0$ в $\mathscr{D}^{\prime}(\Pi)$, где $\theta_{k}=I_{k} / I_{1}$, а при $I_{1}=0$, что равносильно $r(t, x)=0$ (п.в. на П), значения этих функций не играют роли, и можно положить в этом случае $\theta_{k}=0$.

Заметим, что

$$
A \theta_{k}=\int_{\mathbb{R}^{n}} u_{k} d \nu_{t, x}(u), \quad B \theta_{k}=\int_{\mathbb{R}^{n}} \varphi(r) u_{k} d \nu_{t, x}(u)
$$

и из начального условия (55) вытекает соотношение

$$
\underset{t \rightarrow 0+}{\operatorname{ess} \lim _{t}} A \theta_{k}=u_{0 k}=A(0, x) \theta_{0 k}(x) \quad \text { в } L_{\text {loc }}^{1}(\mathbb{R}),
$$

где $\theta_{0 k}(x)=u_{0 k}(x) / u_{01}(x)$. Как следует из условия $(\mathrm{C}), u_{01}(x)=0 \Leftrightarrow u_{0}=0$ п.в. на $\mathbb{R}$, в этом случае можно положить $\theta_{0 k}(x)=0$. Таким образом, компоненты вектор-функции $\theta=\left(\theta_{1}, \ldots, \theta_{n}\right)$ являются (возможно, неограниченньми) о.р. задачи (29), (30) с начальными функциями $\theta_{0 k}$, причем $A \theta_{k}, B \theta_{k} \in L^{\infty}(\Pi)$.

По предложению 6 функция $R=|\theta|$ также является о.р. задачи (29), (30) с начальной функцией $R_{0}=\left|\theta_{0}\right|=r_{0} / u_{01}, \theta_{0}=\left(\theta_{01}, \ldots, \theta_{0 n}\right)$. Тогда $(A R)_{t}+$ $(B R)_{x}=0$ в $\mathscr{D}^{\prime}(\Pi)$, т.е. при $I=I(t, x)=\left(I_{1}, \ldots, I_{n}\right)$

$$
(r|I|)_{t}+(f(r)|I|)_{x}=0 \quad \text { в } \mathscr{D}^{\prime}(\Pi)
$$

Далее, из энтропийного условия (53) с выпуклой энтропией $p(u)=r=|u|$ и потоком $q(u)=f(r)$ следует, что

$$
r_{t}+f(r)_{x} \leqslant 0 \quad \text { в } \mathscr{D}^{\prime}(\Pi)
$$

откуда ввиду (63)

$$
(r(1-|I|))_{t}+(f(r)(1-|I|))_{x} \leqslant 0 \quad \text { в } \quad \mathscr{D}^{\prime}(\Pi) .
$$

По неравенству Йенсена

$$
|I| \leqslant \int_{\mathbb{R}^{n}}|v| d \nu_{t, x}(u) \leqslant 1
$$

откуда следует неотрицательность функции $\alpha=\alpha(t, x)=r(1-|I|)$. При этом

$$
\underset{t \rightarrow 0+}{\operatorname{ess}} \lim _{t \rightarrow 0} r(1-|I|)=r_{0}-\left|u_{0}\right|=0 \quad \text { в } \quad L_{\mathrm{loc}}^{1}(\mathbb{R})
$$

Пусть $\beta=\beta(t, x)=f(r)(1-|I|)$. Тогда из гладкости функции $f$ следует оценка $|\beta| \leqslant$ const $\cdot \alpha$. Далее, ввиду (64) $\alpha_{t}+\beta_{x} \leqslant 0$ в $\mathscr{D}^{\prime}(\Pi)$. Таким образом, функции $\alpha, \beta$ 
удовлетворяют предположениям леммы 4 с $\alpha(0, x) \equiv 0$. По этой лемме $\alpha(t, x)=0$ п.в. на П, и поскольку равенство

$$
(r|I|)^{2}=\left|\int_{\mathbb{R}^{n}} u d \nu_{t, x}(u)\right|^{2}=r^{2}=\int_{\mathbb{R}^{n}}|u|^{2} d \nu_{t, x}(u)
$$

возможно лишь в случае, когда $\nu_{t, x}$ - мера Дирака, мы получаем регулярность мерозначной функции $\nu_{t, x}: \nu_{t, x}(u)=\delta(u-u(t, x))$. По предложению 8 последовательность $u_{k}$ сходится к $u(t, x)$ в $L_{\mathrm{loc}}^{1}\left(\Pi, \mathbb{R}^{n}\right)$. Поскольку условия $(53),(55)$ сводятся в регулярном случае к условиям $(16),(17)$ для $u(t, x)$, то $u(t, x)$ - о.э.р. задачи $(1),(2)$. Далее, по теореме 7 о.э.p. $u(t, x)$ единственно и является сильным. В частности, предельная функция $u(t, x)$ последовательности $u_{k}$ определяется однозначно и не зависит от выбора подпоследовательности. Отсюда следует сходимость исходной последовательности $u_{k}$ (без выделения подпоследовательности). Теорема полностью доказана.

\section{Список литературы}

1. Панов Е. Ю. Об одном классе систем квазилинейных законов сохранения // Матем. сб. 1997. Т. 188. № 5. C. $85-112$.

2. Панов E. Ю. К нелокальной теории обобщенных энтропийных решений задачи Коши для одного класса гиперболических систем законов сохранения // Изв. РАН. Сер. матем. 1999. Т. 63. № 1. С. 133-184.

3. Рождественский Б. Л., Яненко H. Н. Системы квазилинейных уравнений и их приложения к газовой динамике. М.: Наука, 1978.

4. Lax P. D. Hyperbolic systems of conservation laws // Comm. Partial Differential Equations. 1957. V. 10. P. 537-566.

5. Кружков C. Н. Квазилинейные уравнения первого порядка со многими независимьми переменными // Матем. сб. 1970. Т. 81. № 2. С. 228-255.

6. Lax P. D. Shock waves and entropy // Shock waves and entropy, contributions to nonlinear functional analysis / ed. E. A. Zarantonello. New York: Academic Press, 1971. P. 603-634.

7. Хири М. Диффференциальная топология. М.: Мир, 1979.

8. Kruzhkov S. N., Panov E. Yu. Osgood's type conditions for uniqueness of entropy solutions to Cauchy problem for quasilinear conservation laws of the first order // Ann. Univ. Ferrara Sez. VII (N.S.). 1994. V. 15. P. 31-53.

9. Бенилан $\Phi .$, Кружков С. Н. Квазилинейные уравнения первого порядка с непрерьвными нелинейностями // Докл. АН. 1994. Т. 339. № 2. С. 151-154.

10. Benilan Ph., Kruzhkov S. N. Conservation laws with continuous flux functions // NoDEA. Nonlinear Differential Equations Appl. 1996. V. 3. P. 395-419.

11. Панов Е. Ю. О мерозначных решениях задачи Коши для квазилинейного уравнения первого порядка // Изв. РАН. Сер. матем. 1996. Т. 60. № 2. С. 107-148.

12. Кружков C. Н., Панов E. Ю. Консервативные квазилинейные законы первого порядка с бесконечной областью зависимости от начальных данных // Докл. АН СССР. 1990. Т. 314. № 1. C. 79-84.

13. Эйдельман С. Д. Параболические системы. М.: Наука, 1964.

14. Tartar L. Compensated compactness and applications to partial differential equations // Research notes in mathematics, nonlinear analysis, and mechanics. Heriot-Watt Symposium. V. 4, 1979. P. 136-212.

15. DiPerna R. J. Measure-valued solutions to conservation laws // Arch. Rational Mech. Anal. 1985. V. 88. Р. 223-270.

16. Берг Й, Лёфстрём Й. Интерполяционные пространства. М.: Мир, 1980. 
17. Murat F. Compacité par compensation // Ann. Scuola Norm. Sup. Pisa Cl. Sci. (4). 1978. V. 5. P. 489-507.

18. Панов Е. Ю. О сильной предкомпактности ограниченных множеств мерозначных решений квазилинейного уравнения первого порядка // Матем. сб. 1995. Т. 186. № 5. C. $103-114$.

Новгородский государственный университет

E-mail: kafmatan@info.novsu.ac.ru

Поступила в редакцию 03.03.1999 